U.S. Department of the Interior

U.S. Geological Survey

Streamflow and Water-Quality Data for the

Blackfoot River Basin, Western Montana, September 1995 through May 1997

By Sean M. Lawlor

Open-File Report 00-80

In cooperation with MONTANA FISH, WILDLIFE AND PARKS 


\section{U.S. Department of the Interior}

BRUCE BABBITT, Secretary

\section{U.S. Geological Survey}

Charles G. Groat, Director

Any use of trade, product, or firm names in this publication is for descriptive purposes only and does not imply endorsement by the U.S. Government

Helena, Montana

April 2000

For additional information write to:

\section{District Chief}

U.S. Geological Survey

3162 Bozeman Avenue

Helena, MT 59601-6456

Copies of this report may be purchased from:

U.S. Geological Survey

Branch of Information Services

Box 25286

Denver, CO 80225-0286 


\section{CONTENTS}

Abstract

Introduction

Purpose and scope

Site-numbering system

Streamflow and water-quality data

Data-collection methods .

Quality assurance

Water-quality guidelines

Selected references

Data

\section{ILLUSTRATIONS}

Figure 1. Map showing location of the Blackfoot River basin and sampling sites, western Montana

2. Relation between water hardness and freshwater aquatic-life standards for acute and chronic toxicity of selected total-recoverable trace elements

\section{TABLES}

Table 1. Sampling sites in the Blackfoot River basin, western Montana...

2. Streamflow and water-quality data for the Blackfoot River basin, western Montana .

3. Water-quality data for replicate samples from sites in the Blackfoot River basin, western Montana.....

4. Water-quality data for field-blank samples from sites in the Blackfoot River basin, western Montana

5. Montana water-quality maximum guideline concentrations for selected water uses.

\section{CONVERSION FACTORS, VERTICAL DATUM, ABBREVIATED WATER-QUALITY UNITS, AND ACRONYMS}

\begin{tabular}{rll}
\hline Multiply & By & \multicolumn{1}{c}{ To obtain } \\
\hline cubic foot per second $\left(\mathrm{ft}^{3} / \mathrm{s}\right)$ & & \\
$\mathrm{mile}(\mathrm{mi})$ & 0.028317 & cubic meter per second \\
square $\mathrm{mile}\left(\mathrm{mi}^{2}\right)$ & 1.609 & kilometer \\
ton per day (ton/d) & 2.59 & square kilometer \\
kilogram per day \\
ton per acre-foot (ton/acre-ft) & 907.2 & kilogram per cubic meter \\
\hline
\end{tabular}

Temperature can be converted from degrees Celsius $\left({ }^{\circ} \mathrm{C}\right)$ to degrees Fahrenheit $\left({ }^{\circ} \mathrm{F}\right)$ by the following equation:

$$
{ }^{\circ} \mathrm{F}=9 / 5\left({ }^{\circ} \mathrm{C}\right)+32
$$

Sea level: In this report, "sea level" refers to the National Geodetic Vertical Datum of 1929 (NGVD of 1929)--a geodetic datum derived from a general adjustment of the first-order level nets of both the United States and Canada, formerly called Sea Level Datum of 1929.

Water-year definition: A water year is the 12-month period from October 1 through September 30 . It is designated by the calendar year in which it ends.

Chemical concentration in water is reported in milligrams per liter $(\mathrm{mg} / \mathrm{L})$ or micrograms per liter $(\mu \mathrm{g} / \mathrm{L})$. Milligrams per liter is a unit expressing the solute mass (milligrams) per unit volume (liter) of water and is about the same as parts per million unless concentrations are more than 7,000 milligrams per liter (Hem, 1989, p. 55). One thousand micrograms per liter is equivalent to 1 milligram per liter. 
Abbreviated water-quality units used in this report:

col $/ 100 \mathrm{~mL}$ colonies per 100 milliliters

$\mu \mathrm{g} / \mathrm{L} \quad$ microgram per liter

$\mu \mathrm{S} / \mathrm{cm} \quad$ microsiemens per centimeter at 25 degrees Celsius

$\mathrm{mg} / \mathrm{L} \quad$ milligram per liter

$\mathrm{mm} \quad$ millimeter

Acronyms used in this report:

MFWP Montana Fish, Wildlife and Parks

NWQL U.S. Geological Survey National Water Quality Laboratory

USGS U.S. Geological Survey 


\title{
STREAMFLOW AND WATER-QUALITY DATA FOR THE BLACKFOOT RIVER BASIN, WESTERN MONTANA, SEPTEMBER 1995 THROUGH MAY 1997
}

\author{
By Sean M. Lawlor
}

\section{ABSTRACT}

The aquatic resources of the Blackfoot River basin may be affected by natural-resource, agricultural, and residential development. Concern about the effects of past, current, and future activities have resulted in efforts by State, Federal, and private entities to characterize the aquatic resources of the Blackfoot River basin. Baseline data were obtained to describe current conditions and to serve as a reference for future environmental assessments.

This report presents physical, chemical, and biological data from September 1995 through May 1997 for surface-water sites in the Blackfoot River basin. Samples were collected periodically at 13 sites on the Blackfoot River and selected major tributaries. The types of data presented include instantaneous streamflow, onsite measurements of water-quality properties, bacteria, and laboratory analyses for concentrations of chemical constituents and suspended sediment.

\section{INTRODUCTION}

The Blackfoot River originates near Lincoln, in western Montana (fig. 1). Along the 135-mi reach of stream from near the Continental Divide to the Clark Fork near Missoula, six major tributaries enter the Blackfoot River: Alice Creek, Landers Fork, Nevada Creek, North Fork Blackfoot River, Monture Creek, and Clearwater River. Principal surface-water uses in the 2,300 $\mathrm{mi}^{2}$ Blackfoot River basin are irrigation, stock watering, instream flow for aquatic life, waterfowl, and recreation.

Cattle and grain production, logging, mining, and recreation are the primary land uses. Cattle and grain production are the major agricultural land uses in the valley floor, whereas logging and small-scale hardrock mining generally occur in the mountainous parts of the basin. Recreational activities occur throughout the basin, but fishing and boating along the Blackfoot
River are the most common (Duffield and others, 1987).

The Blackfoot River dissects geologically complex, mineralized terranes of the Northern Rocky Mountains (Moore and others, 1991). A variety of metals, including gold, silver, lead, and copper, have been mined in this area from numerous small placer and hard-rock mining operations. The basin is extensively glaciated, with glacial deposits extending from the surrounding mountains into the valley floor. Perennial and seasonal wetlands are present in potholes of the valley glacial deposits and provide nesting sites for the area's abundant waterfowl, as well as habitat for a variety of plant species. Coniferous forests and upland grasses predominate in the mountains and foothills, whereas interspersed grasses, sagebrush, conifers, and deciduous riparian communities are found on the valley floor.

The Blackfoot River is classified as a Class I trout stream by Montana Fish, Wildlife and Parks. The trout fishery is a complex, multispecies assemblage (Spence, 1975; Peters and Spoon, 1989). In general, eastern brook trout and cutthroat trout are the dominant species in the headwater reaches. Brown trout predominate in the middle reaches and rainbow trout predominate in the lower reaches. Bull trout also are found in the middle and lower reaches of the river. The benthic community in much of the Blackfoot River basin is a diverse assemblage of insect larvae typical of temperate, cobble-bed rivers (Spence, 1975).

The aquatic resources of the Blackfoot River basin may be affected by natural-resource, agricultural, and residential development. Mining and logging have resulted in localized water-quality degradation and stream sedimentation (Moore and others, 1991). Livestock grazing, irrigation, and agricultural chemical applications have resulted in riparian and range degradation, dewatering, and nutrient enrichment of streams (Rothrock and others, 1998). 


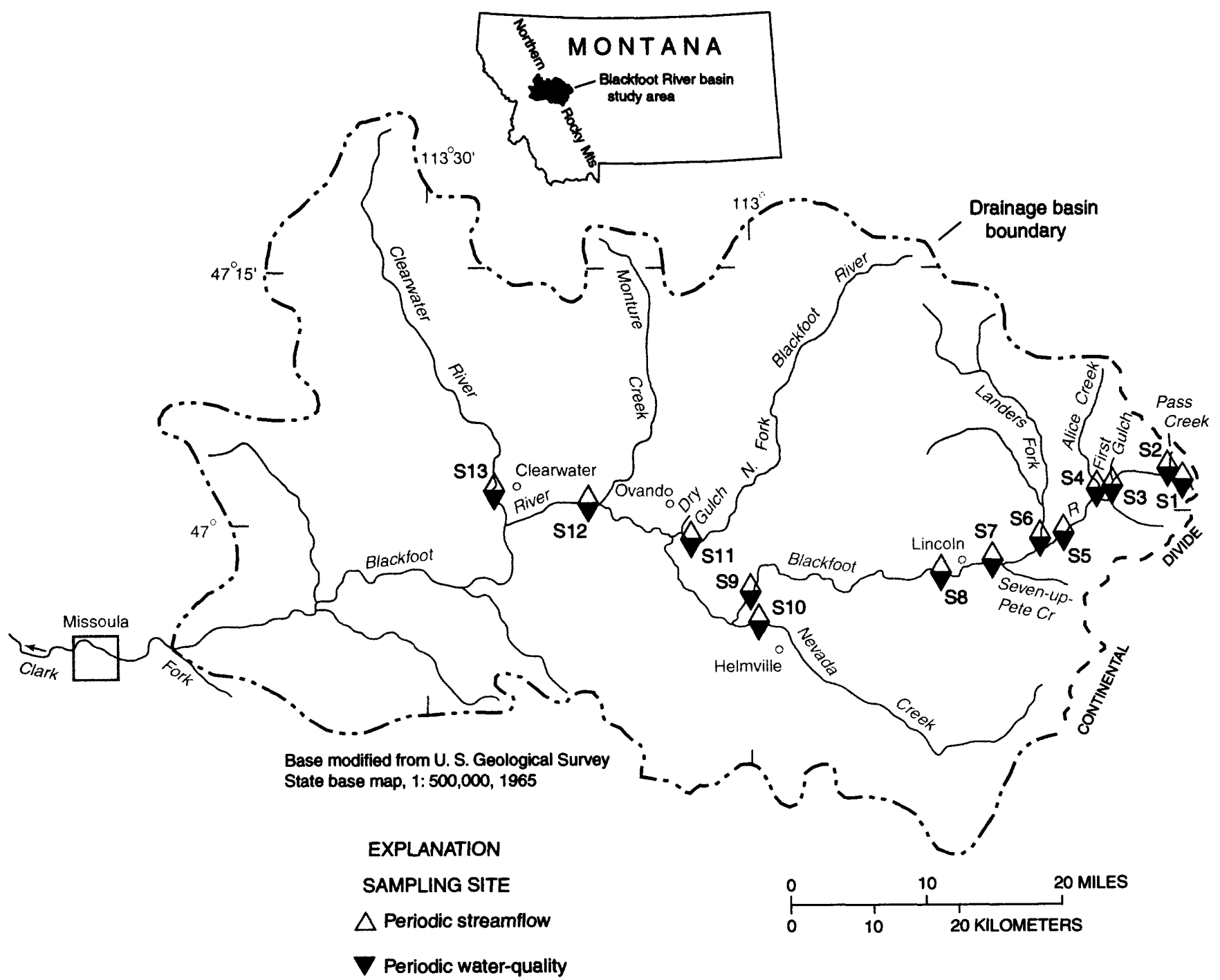

S13 SITE NUMBER

Figure 1. Location of the Blackfoot River basin and sampling sites, western Montana.

Residential development and associated septic systems near stream corridors can affect the quality of shallow ground water and surface water. Elevated trace-element concentrations in bed sediment derived from historical mining in the headwaters of the Blackfoot River extend farther downstream than elevated trace-element concentrations in water. This sediment enriched with trace elements will likely continue to migrate downstream and may affect aquatic life (Moore and others, 1991).

Concern about the effects of past, current, and future activities have resulted in efforts by State, Federal, and private entities to characterize the aquatic resources of the Blackfoot River basin. Baseline data were needed to describe current conditions and to serve as a reference for future environmental assessments. Consequently, the U.S. Geological Survey (USGS), in cooperation with Montana Fish, Wildlife and Parks (MFWP), initiated a two-year effort to obtain streamflow and water-quality data for the Blackfoot River and selected tributaries.

\section{Purpose and Scope}

The purpose of this report is to present physical, chemical, and biological data collected from 13 surface-water sampling sites (table 1) in the Blackfoot 
River basin during the period September 1995 through May 1997. Samples were collected periodically at a prorated frequency of four times per year. Data presented in this report include measurements of streamflow quantity and quality, and quality-assurance data.

\section{Site-Numbering System}

To simplify site identification, surface-water sites are assigned a site number from $\mathrm{S} 1$ to $\mathrm{S} 13$ (fig. 1; table 1). In addition, each site is assigned an eight-digit or fifteen-digit station-identification number. Eightdigit station-identification numbers for surface-water sites represent the standard USGS numbering system for streamflow-gaging stations or routine streamflowmeasurement sites. Fifteen-digit station-identification numbers are used for miscellaneous or temporary surface-water stations. The fifteen-digit numbers represent the approximate latitude and longitude of the site (first 13 digits), plus the sequence number (last 2 digits).

\section{STREAMFLOW AND WATER- QUALITY DATA}

Streamflow was measured and water-quality samples were collected periodically over two years on the mainstem of the Blackfoot River and selected tributaries. Samples were collected at a prorated frequency of four times per year, with sampling times distributed seasonally in order to characterize the hydrologic vari- ability of the quantity and quality of the surface water of the Blackfoot River basin. The types of data collected for all sites include instantaneous streamflow, onsite measurements of water-quality properties (specific conductance, $\mathrm{pH}$, and air and water temperature), and laboratory analytical results for chemical constituents and suspended sediment. At selected sites, dissolved oxygen and bacteria (fecal coliform and fecal streptococci) concentrations also were measured onsite. Streamflow and water-quality data are presented in table 2 (tables 2-5 at back of report).

The quality of data was maintained through documented procedures designed to provide environmentally representative data. Acceptable performance of the procedures was verified with quality-control samples that were collected systematically to provide a measure of the accuracy, precision, and bias of the environmental data and to identify problems associated with sample collection, processing, or analysis. These quality-control data are presented in tables 3 and 4 .

\section{Data-Collection Methods}

Instantaneous streamflow at the time of sampling was determined from either a stage-discharge relation or by discharge measurement. Discharge measurements were made according to established USGS procedures as outlined in Rantz and others (1982).

Cross-sectional water samples for analysis of chemical constituents and suspended sediment were

Table 1. Sampling sites in the Blackfoot River basin, western Montana

[Site number shown in figure 1 and described in text. Station number described in text]

\begin{tabular}{cll}
\hline Site number & \multicolumn{1}{c}{ Station number } & \multicolumn{1}{c}{ Station name } \\
\hline S1 & 470226112224501 & Blackfoot River above Pass Creek, near Lincoln, Montana \\
S2 & 470314112223901 & Pass Creek near Lincoln, Montana \\
S3 & 12334620 & Blackfoot River below First Gulch, near Lincoln, Montana \\
S4 & 470114112280701 & Alice Creek near Lincoln, Montana \\
S5 & 12334650 & Blackfoot River below Alice Creek, near Lincoln, Montana \\
S6 & 12334680 & Landers Fork near Lincoln, Montana \\
S7 & 12334700 & Blackfoot River below Seven-up-Pete Creek, near Lincoln, Montana \\
S8 & 12334800 & Blackfoot River at Dalton Mountain Road bridge, near Lincoln, Montana \\
S9 & 12335100 & Blackfoot River above Nevada Creek, near Helmville, Montana \\
S10 & 465330113021601 & Nevada Creek at mouth, near Helmville, Montana \\
S11 & 12338300 & North Fork Blackfoot River above Dry Gulch, near Ovando, Montana \\
S12 & 12338700 & Blackfoot River at Scotty Brown Bridge, near Ovando, Montana \\
S13 & 12339450 & Clearwater River near Clearwater, Montana \\
\hline
\end{tabular}


collected from multiple verticals across the stream using depth- and width-integration methods described by Knapton (1985) and Edwards and Glysson (1988). These methods provide a vertically and laterally discharge-weighted sample that is representative of the entire flow through the cross section of a stream. Sampling equipment consisted of standard USGS depthintegrating samplers (DH-81, DH-48TM, and D74TM), which are either constructed of plastic or coated with a non-metallic epoxy paint, and equipped with nylon nozzles that can be interchanged depending on flow conditions.

Onsite measurements of specific conductance, $\mathrm{pH}$, air and water temperature, and dissolved oxygen were made during collection of periodic water-quality samples. Onsite sample processing, including sample splitting, filtration, and preservation, was performed according to procedures described by Horowitz and others (1994), Ward and Harr (1990), and Knapton (1985). Water samples for bacteria were cultured and enumerated in the field according to Knapton (1985).

Water samples were analyzed for selected common ions, nutrients, and trace elements by the USGS National Water Quality Laboratory (NWQL) in Arvada, Colorado. Common ions and nutrients were analyzed for dissolved concentrations, whereas arsenic, cadmium, copper, iron, lead, manganese, and zinc were analyzed for total-recoverable concentrations. Analytical methods for chemical constituents are described by Fishman and Friedman (1989) and Fishman (1993).

Cross-sectional water samples also were collected for analysis of suspended sediment whenever periodic water-quality samples were collected. These samples were analyzed for suspended-sediment concentration and the percentage of suspended sediment finer than $0.062-\mathrm{mm}$ diameter (silt size and smaller) by the USGS sediment laboratory in Helena, Mont., according to methods described by Guy (1969) and Lambing and Dodge (1993).

\section{Quality Assurance}

Quality-assurance procedures used for the collection and field processing of water-quality samples are described by Horowitz and others (1994), Ward and Harr (1990), Edwards and Glysson (1988), Knapton and Nimick (1991), and Knapton (1985). Standard procedures used by the NWQL for internal sample handling and quality assurance are described by Friedman and Erdmann (1982), Jones (1987), and Pritt and Raese (1992). Quality-assurance procedures used for the collection and processing of suspended-sediment samples by the Montana District are described by Lambing and Dodge (1993).

The quality of analytical results reported for water-quality samples was evaluated by quality-control samples that were submitted from the field and analyzed concurrently in the laboratory with routine samples. These quality-control samples consisted of replicates and field blanks which provide quantitative information on the precision and bias of the overall field and laboratory process. Each type of quality-control sample was submitted at a proportion equivalent to about 6 percent of the total number of water-quality samples; therefore, the total number of quality-control samples represented about 13 percent of the total number of water-quality samples.

In addition to quality-control samples submitted from the field, internal quality-assurance practices at the NWQL are performed systematically to provide quality control of analytical procedures (Pritt and Raese, 1992). These internal practices include analyses of quality-control samples such as calibration standards, standard reference water samples, replicate samples, deionized-water blanks, or spiked samples at a proportion equivalent to at least 10 percent of the sample load. The NWQL participates in a blind-sample program where standard reference water samples prepared by the USGS Branch of Quality Systems are routinely inserted into the sample line for each analytical method at a frequency proportional to the sample load. The laboratory also participates in evaluation studies twice-yearly with the U.S. Environmental Protection Agency, the Canadian Center for Inland Water, and the Branch of Quality Systems to assess analytical performance.

Replicate data can be obtained in different ways to provide an assessment of precision (reproducibility) of analytical results. Replicate samples are two or more samples considered to be essentially identical in composition. Replicate field samples can be obtained by either repeating the collection process to obtain two or more samples or by splitting a single sample into two or more subsamples, which are then analyzed separately. Likewise, a single sample can be analyzed two 
or more times in the laboratory to obtain a measure of analytical precision.

Precision of analytical results for field replicates is affected by numerous sources of variability within the field and laboratory environments, including sample collection, sample processing, and sample analysis. To provide data on overall reproducibility of results for samples exposed to all sources of variability, replicate samples were obtained in the field by splitting a composite stream sample. Paired analyses of field replicate samples (table 3) were used to estimate precision for each parameter, expressed as a relative standard deviation, in percent. Values less than the minimum reporting level were treated as being equal to the minimum reporting level for calculating the relative standard deviation. Relative standard deviations estimated from differences in the paired analytical results for field replicates were typically within 10 percent for most parameters, and all were within 20 percent, except nitrite (dissolved) and arsenic (total recoverable).

Because nitrite and arsenic concentrations in the replicates were all near or less than the minimum reporting level, the relative standard deviation in excess of 20 percent is considered to be insignificant owing to the exaggerated effect of small differences when expressed in units of percent.

Blank samples of deionized water were routinely analyzed to identify the presence and magnitude of contamination that potentially could bias analytical results. The particular type of blank sample tested was a "field" blank. Field blanks are aliquots of deionized water that are certified as trace-element free and are processed through the same sampling equipment used to collect stream samples. These blanks are then subjected to the same processing (sample splitting, filtration, preservation, transportation, and laboratory handling) as stream samples. Blank samples are analyzed for the same constituents as those of stream samples to identify whether any detectable concentrations exist. Results of analyses of blank samples are presented in table 4. Almost all concentrations in blanks were less than the minimum reporting levels and none of the few detectable concentrations were greater than twice the minimum reporting level. Therefore, the sampling and analytical process was essentially free of any significant contamination bias.

\section{Water-Quality Guidelines}

General information is provided in this section to enable the reader to compare various water-quality guidelines to the constituent concentrations measured in the Blackfoot River basin during 1995-97 (table 2). The Montana water-quality maximum guideline concentrations for selected water uses are shown in table 5 . Figure 2 graphically illustrates the hardness-dependent variation in freshwater aquatic-life standards for acute and chronic toxicity for selected trace elements. 

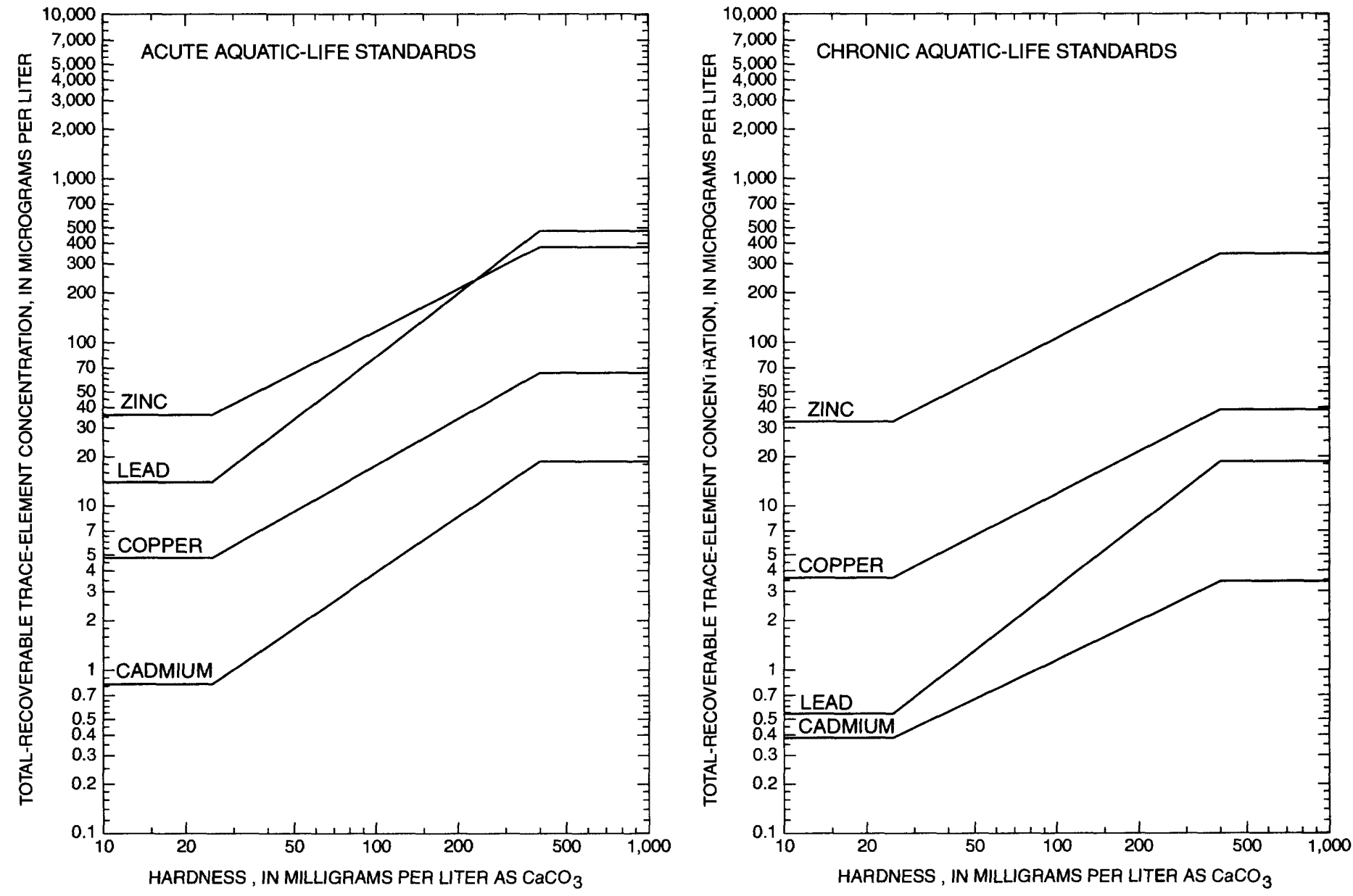

Figure 2. Relation between water hardness and freshwater aquatic-life standards for acute and chronic toxicity of selected totalrecoverable trace elements (Montana Department of Environmental Quality, 1995).

\section{SELECTED REFERENCES}

Axtmann, E.V., and Luoma, S.N., 1991, Large scale distribution of metal contamination in the fine-grained sediment of the Clark Fork River, Montana: Applied Geochemistry, v. 6, p. 75-88.

Britton, L.J., and Greeson, P.E., eds., 1989, Methods for collection and analysis of aquatic biological and microbiological samples: U.S. Geological Survey Techniques of Water-Resources Investigations, book 5 , chap. A4, 363 p.

Cain, D.J., Luoma, S.N., Carter, J.L., and Ferd, S.V., 1992, Aquatic insects as bioindicators of trace element contamination in cobble-bottom rivers and streams: Canadian Journal of Fisheries and Aquatic Sciences, v. 49 , no. 10 , p. $2141-2154$.
Coffin, D.L., and Wilke, K.R., 1971, Water resources of the upper Blackfoot River valley, west-central Montana: Helena, Montana Department of Natural Resources and Conservation, Water Resources Division Technical Report Series 1, 82 p.

Dodge, K.A., Hornberger, M.I., and Axtmann, E.V., 1998, Water-quality, bed sediment, and biological data (October 1996 through September 1997) and statistical summaries of data for streams in the upper Clark Fork basin, Montana: U.S. Geological Survey Open-File Report 98-407, 102 p.

Duffield, John, Loomis, John, and Brooks, Rob, 1987, The net economic value of fishing in Montana: Helena, Montana Department of Fish, Wildlife and Parks, 45 p.

Edwards, T.K., and Glysson, G.D., 1988, Field methods for measurement of fluvial sediment: U.S. Geological Survey Open-File Report 86-531, 118 p. 
Fishman, M.J., 1993, Methods of analysis by the U.S. Geological Survey National Water Quality Laboratory --Determination of inorganic and organic constituents in water and fluvial sediments: U.S. Geological Survey Open-File Report 93-125, 217 p.

Fishman, M.J., and Friedman, L.C., eds., 1989, Methods for determination of inorganic substances in water and fluvial sediments: U.S. Geological Survey Techniques of Water-Resources Investigations, book 5, chap. A1, $545 \mathrm{p}$.

Friedman, L.C., and Erdmann, D.E., 1982, Quality assurance practices for the chemical and biological analyses of water and fluvial sediments: U.S. Geological Survey Techniques of Water-Resources Investigations, book 5 , chap. A6, 181 p.

Guy, H.P., 1969, Laboratory theory and methods for sediment analysis: U.S. Geological Survey Techniques of Water-Resources Investigations, book 5, chap. C1, $58 \mathrm{p}$.

Hem, J.D., 1989, Study and interpretation of the chemical characteristics of natural water ( $3 d$ ed.): U.S.

Geological Survey Water-Supply Paper 2254, 263 p.

Horowitz, A.J., Demas, C.R., Fitzgerald, K.K., Miller, T.L., and Rickert, D.A., 1994, U.S. Geological Survey protocol for the collection and processing of surfacewater samples for the subsequent determination of inorganic constituents in filtered water: U.S. Geological Survey Open-File Report 94-539, 57 p.

Ingman, G.L., Kerr, M.A., and McGuire, D.L, 1990, Water quality investigations in the Blackfoot River drainage, Montana: Helena, Montana Department of Health and Environmental Sciences, Water Quality Bureau, 118 p.

Jones, B.E., 1987, Quality control manual of the U.S. Geological Survey's National Water Quality Laboratory: U.S. Geological Survey Open-File Report $87-457,17 \mathrm{p}$.

Knapton, J.R., 1985, Field guidelines for collection, treatment, and analysis of water samples, Montana District: U.S. Geological Survey Open-File Report 85409, $86 \mathrm{p}$.

Knapton, J.R., and Nimick, D.A., 1991, Quality assurance for water-quality activities of the U.S. Geological Survey in Montana: U.S. Geological Survey Open-File Report 91-216, 41 p.

Lambing, J.H., and Dodge, K.A., 1993, Quality assurance for laboratory analysis of suspended-sediment samples by the U.S. Geological Survey in Montana: U.S. Geological Survey Open-File Report 93-131, 34 p.

Meador, M.R., Hupp, C.H., Cuffney, T.F., and Gurtz, M.E.,1993, Methods for characterizing stream habitat as part of the National Water-Quality Assessment Program: U.S. Geological Survey Open-File Report 93-408, $48 \mathrm{p}$.
Menges, Jennifer, 1997, Investigation of temporal changes of heavy metal concentrations in sediments and water of the Blackfoot River: Missoula, University of Montana, M.S. thesis, $163 \mathrm{p}$.

Montana Department of Environmental Quality, 1995, Montana numeric water quality standards: Helena, Mont., Water Quality Division, Circular WQB-7, 39 p.

Montana Department of Health and Environmental Sciences, 1986, Montana water quality, Montana 305(b) Report: Helena, Mont, 198 p.

Moore, J.N., Luoma, S.N., and Peters, D., 1991, Downstream effects of mine effluent on an intermontane riparian system: Canadian Journal of Fisheries and Aquatic Sciences, v. 48 , no. 2 , p. 222-231.

Peters, Don, and Spoon, Ron, 1989, Preliminary fisheries inventory of the Big Blackfoot River: Helena, Montana Department of Fish, Wildlife and Parks, 46 p.

Peters, Don, 1990, Inventory of fishery resources in the Blackfoot River and major tributaries: Helena, Montana Department of Fish, Wildlife and Parks, $60 \mathrm{p}$.

Pierce, Ron, 1991, A stream habitat and fisheries analysis for six tributaries to the Big Blackfoot River: Helena, Montana Department of Fish, Wildlife and Parks, 41 p.

Pierce, Ron, and Peters, Don, 1990, Aquatic investigations in the middle Blackfoot River, Nevada Creek, and Nevada Spring Creek corridor: Helena, Montana Department of Fish, Wildlife and Parks, 22 p.

Pritt, J.W., and Raese, J.W., eds., 1992, Quality assurance/ quality control manual--National Water Quality Laboratory: U.S. Geological Survey Open-File Report 92-495, $33 \mathrm{p}$.

Rantz, S.E., and others, 1982, Measurement and computation of streamflow: U.S. Geological Survey Water-Supply Paper 2175, 2 v., 631 p.

Rothrock, J.A., Barten, P.K., and Ingman, G.L., 1998, Land use and aquatic biointegrity in the Blackfoot River watershed, Montana: Journal of the American Water Resources Association, v. 34, no. 3, p. 565-581.

Spence, L.E., 1975, Upper Blackfoot River study--a premining inventory of aquatic and wildlife resources: Helena, Montana Department of Fish and Game, multilith, $85 \mathrm{p}$.

Ward, J.R., and Harr, C.A., eds., 1990, Methods for collection and processing of surface-water and bedmaterial samples for physical and chemical analyses: U.S. Geological Survey Open-File Report 90-140, 71 p.

Wilke, K.R., 1976, Ground-water levels and chemical quality of ground water in Lincoln, Montana: U.S. Geological Survey Open-File Report 76-333, 4 sheets.

Wilke, K.R., 1979, Selected well inventory and chemical analyses of ground water, parts of Missoula and Powell Counties, Montana: U.S. Geological Survey Open-File Report 79-1491, 4 leaves. 
8 Streamflow and water-quality data for the Blackfoot River Basin, Western Montana, September 1995 through May 1997 
DATA

DATA 9 
Table 2. Streamflow and water-quality data for the Blackfoot River basin, western Montana

[Site number shown in figure 1 and described in text. Abbreviations: $\mathrm{ft}^{3} / \mathrm{s}$, cubic feet per second; $\mu \mathrm{S} / \mathrm{cm}$, microsiemens per centimeter at $25^{\circ} \mathrm{C}$; ${ }^{\circ} \mathrm{C}$, degrees Celsius; $\mathrm{mg} / \mathrm{L}$, milligrams per liter; col/100mL, colonies per 100 milliiters; $\mathrm{K}$, results based on non-ideal colony count; ton/acre-ft, tons per acre-foot; ton/d, tons per day; $\mu \mathrm{g} / \mathrm{L}$, micrograms per liter; mm, millimeter. Symbols: <, less than minimum reporting level; --, no data]

\begin{tabular}{|c|c|c|c|c|c|c|c|c|c|c|}
\hline $\begin{array}{c}\text { Site } \\
\text { number }\end{array}$ & Date & Time & $\begin{array}{c}\text { Streamflow, } \\
\text { instantaneous } \\
\left(\mathrm{ft}^{3} / \mathrm{s}\right)\end{array}$ & $\begin{array}{c}\text { Specific } \\
\text { conductance, } \\
\text { onsite } \\
(\mu \mathrm{S} / \mathrm{cm})\end{array}$ & $\begin{array}{c}\text { pH, } \\
\text { onsite } \\
\text { (standard } \\
\text { units) }\end{array}$ & $\begin{array}{c}\text { Tempera- } \\
\text { ture, } \\
\text { air } \\
\left({ }^{\circ} \mathrm{C}\right)\end{array}$ & $\begin{array}{c}\text { Tempera- } \\
\text { ture, } \\
\text { water } \\
\left({ }^{\circ} \mathrm{C}\right)\end{array}$ & $\begin{array}{c}\text { Oxygen, } \\
\text { dissolved, } \\
\text { onsite } \\
\text { (mg/L) }\end{array}$ & $\begin{array}{c}\text { Oxygen, } \\
\text { dissolved, } \\
\text { onsite } \\
\text { (percent } \\
\text { saturation) }\end{array}$ & $\begin{array}{c}\text { Coliform, } \\
\text { fecal } \\
\text { (colv100 } \\
\text { mL) }\end{array}$ \\
\hline \multirow[t]{8}{*}{ SI } & $09-07-95$ & 1250 & 2.8 & 293 & 7.5 & 9.5 & 10.0 & -- & -- & - \\
\hline & $11-02-95$ & 0920 & 2.4 & 304 & 7.4 & -10.0 & 2.0 & -- & - & - \\
\hline & $04-19-96$ & 1230 & 28 & 192 & 7.8 & 6.0 & 4.5 & - & - & -- \\
\hline & $06-20-96$ & 1500 & 13 & 300 & 7.7 & 9.0 & 14.5 & - & -- & -- \\
\hline & $08-21-96$ & 1400 & 2.4 & 242 & 8.0 & 20.0 & 14.5 & -- & - & - \\
\hline & $10-24-96$ & 1250 & 1.2 & 321 & 7.4 & 3.0 & 4.0 & -- & - & - \\
\hline & $04-14-97$ & 1100 & 3.0 & 410 & 7.4 & 2.0 & 2.0 & -- & - & - \\
\hline & $05-30-97$ & 1620 & 52 & 146 & 7.7 & 20.5 & 9.0 & -- & $\cdots$ & - \\
\hline \multirow[t]{8}{*}{ S2 } & $09-07-95$ & 1130 & .38 & 165 & 7.9 & 7.5 & 9.5 & - & - & - \\
\hline & $11-02-95$ & 0810 & .19 & 159 & 7.6 & -13.0 & 1.0 & -- & -- & -- \\
\hline & $04-19-96$ & 1100 & 7.9 & 111 & 8.1 & 4.0 & 3.0 & -- & - & - \\
\hline & $06-20-96$ & 1330 & 1.5 & 137 & 8.1 & 16.0 & 9.0 & -- & -- & -- \\
\hline & $08-21-96$ & 1310 & .20 & 162 & 8.1 & 18.0 & 11.0 & -- & -. & - \\
\hline & $10-24-96$ & 1200 & .19 & 158 & 7.8 & 3.0 & 5.0 & - & - & -- \\
\hline & $04-14-97$ & 1200 & .41 & 159 & 7.9 & 3.0 & 2.5 & -- & - & - \\
\hline & $05-30-97$ & 1510 & 8.2 & 107 & 7.9 & 21.0 & 10.0 & - & -- & -- \\
\hline \multirow[t]{8}{*}{ S3 } & $09-07-95$ & 1030 & 6.0 & 232 & 8.0 & 13.0 & 12.5 & -- & $\ldots$ & -- \\
\hline & $11-02-95$ & 1020 & 8.1 & 228 & 7.8 & -6.0 & 0.0 & -- & -. & -- \\
\hline & $04-19-96$ & 1000 & 99 & 163 & 7.8 & 3.0 & 2.0 & -. & -- & -- \\
\hline & $06-20-96$ & 1230 & 26 & 233 & 8.0 & 16.0 & 10.0 & -- & - & -. \\
\hline & $08-21-96$ & 1200 & 5.6 & 226 & 8.0 & 16.0 & 13.0 & -- & - & -- \\
\hline & $10-24-96$ & 1100 & 4.5 & 219 & 7.7 & 1.5 & 1.5 & -- & - & - \\
\hline & $04-14-97$ & 1300 & 7.8 & 245 & 7.7 & 4.5 & 1.5 & - & -. & -. \\
\hline & $05-30-97$ & 1415 & 113 & 154 & 7.9 & 22.5 & 12.5 & -- & -. & -. \\
\hline \multirow[t]{8}{*}{ S4 } & $09-07-95$ & 0920 & 11 & 236 & 8.2 & 10.5 & 10.5 & - & - & - \\
\hline & $11-02-95$ & 1200 & 6.1 & 243 & 7.9 & -2.0 & 2.5 & - & - & - \\
\hline & $04-19-96$ & 0845 & 102 & 167 & 7.9 & 1.0 & 2.0 & - & - & - \\
\hline & $06-20-96$ & 0815 & 48 & 206 & 8.2 & 3.0 & 6.5 & -- & - & -- \\
\hline & $08-21-96$ & 1040 & 12 & 239 & 8.2 & 12.5 & 10.0 & -- & - & -- \\
\hline & $10-24-96$ & 1015 & 9.0 & 237 & 8.0 & 1.0 & 2.5 & - & - & -. \\
\hline & $04-14-97$ & 1410 & 8.6 & 220 & 8.2 & 5.5 & 6.5 & - & - & -- \\
\hline & $05-30-97$ & 1315 & 131 & 168 & 8.2 & 22.5 & 9.5 & -- & - & -- \\
\hline \multirow[t]{8}{*}{ S5 } & $09-07-95$ & 0830 & 19 & 239 & 8.3 & 11.0 & 9.5 & -. & - & -. \\
\hline & $11-02-95$ & 1430 & 19 & 238 & 8.5 & .0 & 0.0 & -- & - & - \\
\hline & $04-18-96$ & 1640 & 320 & 156 & 8.2 & 4.0 & 4.0 & -- & -- & -- \\
\hline & $06-20-96$ & 0930 & 96 & 201 & 8.1 & 8.0 & 8.0 & -- & - & -- \\
\hline & $08-21-96$ & 0920 & 25 & 236 & 8.4 & 14.5 & 9.5 & -- & - & -- \\
\hline & $10-24-96$ & 0920 & 18 & 241 & 8.2 & .5 & 3.0 & -- & -- & - \\
\hline & $04-14-97$ & 1520 & 28 & 232 & 8.4 & 5.0 & 6.5 & -- & - & -- \\
\hline & $05-30-97$ & 1200 & 316 & 156 & 8.1 & 20.5 & 10.5 & -- & - & -- \\
\hline
\end{tabular}


Table 2. Streamflow and water-quality data for the Blackfoot River basin, western Montana (Continued)

\begin{tabular}{|c|c|c|c|c|c|c|c|c|c|c|c|}
\hline $\begin{array}{c}\text { Site } \\
\text { number }\end{array}$ & Date & $\begin{array}{l}\text { Streptococci, } \\
\text { fecal } \\
(\mathrm{col} / 100 \mathrm{~mL})\end{array}$ & $\begin{array}{c}\text { Hard- } \\
\text { ness, } \\
\text { total } \\
(\mathrm{mg} / \mathrm{L} \text { as } \\
\left.\mathrm{CaCO}_{3}\right) \\
\end{array}$ & $\begin{array}{l}\text { Calcium, } \\
\text { dissolved } \\
(\mathrm{mg} / \mathrm{L} \text { as } \\
\text { Ca) }\end{array}$ & $\begin{array}{l}\text { Magne- } \\
\text { sium, } \\
\text { dissolved } \\
\text { (mg/L as } \\
\text { Mg) }\end{array}$ & $\begin{array}{l}\text { Sodium, } \\
\text { dissolved } \\
\text { (mg/L as } \\
\mathrm{Na})\end{array}$ & $\begin{array}{l}\text { Sodium } \\
\text { adsorption } \\
\text { ratio }\end{array}$ & $\begin{array}{c}\text { Potassium, } \\
\text { dissolved } \\
\text { (mg/L as } \\
\mathrm{K})\end{array}$ & $\begin{array}{c}\text { Alkalinity, } \\
\text { laboratory } \\
(\mathrm{mg} / \mathrm{L} \text { as } \\
\left.\mathrm{CaCO}_{3}\right)\end{array}$ & $\begin{array}{c}\text { Sulfate, } \\
\text { dissolved } \\
(\mathrm{mg} / \mathrm{L} \text { as } \\
\left.\mathrm{SO}_{4}\right)\end{array}$ & $\begin{array}{c}\text { Chlo- } \\
\text { ride, } \\
\text { dissolved } \\
\text { (mg/L as } \\
\mathrm{Cl} \text { ) }\end{array}$ \\
\hline \multirow[t]{8}{*}{ S1 } & $09-07-95$ & -- & 140 & 28 & 16 & 1.5 & 0.1 & 0.8 & 58 & 84 & 0.2 \\
\hline & $11-02-95$ & -- & 140 & 29 & 16 & 1.6 & .1 & .8 & 46 & 98 & .2 \\
\hline & $04-19-96$ & -- & 87 & 19 & 9.7 & 1.2 & .1 & .5 & 43 & 51 & .2 \\
\hline & $06-20-96$ & -- & 140 & 29 & 17 & 1.3 & $<.1$ & .5 & 57 & 90 & .1 \\
\hline & $08-21-96$ & -- & 110 & 24 & 13 & 1.4 & .1 & .7 & 63 & 56 & $<.1$ \\
\hline & $10-24-96$ & -- & 150 & 31 & 17 & 1.7 & .1 & .6 & 56 & 100 & $<.1$ \\
\hline & $04-14-97$ & -- & 200 & 41 & 23 & 1.6 & .1 & .7 & 53 & 150 & .3 \\
\hline & $05-30-97$ & -- & 66 & 15 & 7.2 & .9 & $<.1$ & 4 & 50 & 20 & .2 \\
\hline \multirow[t]{8}{*}{ S2 } & $09-07-95$ & -- & 78 & 19 & 7.5 & 2.2 & .1 & .5 & 83 & 4.4 & 1.1 \\
\hline & $11-02-95$ & -- & 78 & 19 & 7.3 & 2.1 & .1 & .4 & 76 & 6.1 & 1.5 \\
\hline & $04-19-96$ & -- & 53 & 13 & 5.1 & 1.9 & .1 & .4 & 54 & 3.2 & 1.2 \\
\hline & $06-20-96$ & -- & 67 & 16 & 6.5 & 1.8 & .1 & .3 & 69 & 3.1 & .5 \\
\hline & $08-21-96$ & - & 80 & 20 & 7.4 & 2.0 & .1 & .5 & 81 & 4.5 & .7 \\
\hline & $10-24-96$ & - & 77 & 19 & 7.2 & 2.1 & .1 & .5 & 77 & 6.3 & 1.0 \\
\hline & $04-14-97$ & - & 76 & 18 & 7.3 & 2.6 & .1 & .4 & 68 & 6.4 & 4.8 \\
\hline & $05-30-97$ & -- & 48 & 12 & 4.5 & 1.5 & .1 & .3 & 51 & 2.4 & .9 \\
\hline \multirow[t]{8}{*}{ S3 } & $09-07-95$ & - & 110 & 24 & 12 & 1.8 & .1 & .8 & 85 & 32 & .4 \\
\hline & $11-02-95$ & -- & 110 & 24 & 12 & 1.9 & .1 & .7 & 73 & 36 & .6 \\
\hline & $04-19-96$ & - & 80 & 18 & 8.4 & 1.4 & .1 & .6 & 61 & 21 & .5 \\
\hline & $06-20-96$ & -- & 120 & 25 & 13 & 1.5 & .1 & .4 & 67 & 49 & .3 \\
\hline & $08-21-96$ & -- & 110 & 25 & 11 & 1.7 & .1 & .7 & 89 & 27 & .4 \\
\hline & $10-24-96$ & -- & 100 & 23 & 11 & 1.9 & .1 & .7 & 71 & 41 & .3 \\
\hline & $04-14-97$ & -- & 120 & 26 & 13 & 1.9 & .1 & .9 & 65 & 54 & 1.2 \\
\hline & $05-30-97$ & -- & 69 & 16 & 7.3 & 1.2 & .1 & .4 & 59 & 16 & .3 \\
\hline \multirow[t]{8}{*}{$\$ 4$} & $09-07-95$ & -- & 120 & 28 & 12 & 1.9 & .1 & .7 & 126 & 2.7 & .2 \\
\hline & $11-02-95$ & -- & 120 & 30 & 12 & 2.0 & .1 & .7 & 129 & 3.2 & .4 \\
\hline & $04-19-96$ & -- & 86 & 21 & 8.1 & 1.5 & .1 & .6 & 88 & 3.1 & .3 \\
\hline & $06-20-96$ & - & 100 & 25 & 10 & 1.7 & .1 & .6 & 109 & 2.9 & .2 \\
\hline & $08-21-96$ & -- & 120 & 30 & 11 & 1.8 & .1 & .7 & 128 & 2.5 & .2 \\
\hline & $10-24-96$ & -- & 120 & 29 & 12 & 2.0 & .1 & .6 & 128 & 3.2 & .2 \\
\hline & $04-14-97$ & -- & 110 & 26 & 11 & 2.0 & .1 & .6 & 114 & 3.8 & .4 \\
\hline & $05-30-97$ & -- & 83 & 20 & 7.8 & 1.4 & .1 & .5 & 86 & 2.2 & .3 \\
\hline \multirow[t]{8}{*}{ S5 } & 09-07-95 & -- & 120 & 28 & 12 & 2.3 & .1 & .8 & 116 & 11 & .6 \\
\hline & $11-02-95$ & -- & 120 & 29 & 12 & 2.5 & .1 & .9 & 117 & 12 & .6 \\
\hline & $04-18-96$ & -- & 76 & 18 & 7.6 & 1.6 & .1 & .7 & 72 & 10 & .5 \\
\hline & $06-20-96$ & -- & 100 & 24 & 10 & 1.9 & .1 & .6 & 91 & 14 & .5 \\
\hline & $08-21-96$ & -- & 120 & 28 & 11 & 2.4 & .1 & .9 & 118 & 9.1 & .5 \\
\hline & $10-24-96$ & -. & 120 & 29 & 12 & 2.7 & .1 & .9 & 120 & 12 & .3 \\
\hline & $04-14-97$ & -- & 120 & 27 & 12 & 2.3 & .1 & .9 & 103 & 17 & .8 \\
\hline & $05-30-97$ & -- & 73 & 17 & 7.2 & 1.5 & .1 & .6 & 72 & 7.7 & .3 \\
\hline
\end{tabular}


Table 2. Streamflow and water-quality data for the Blackfoot River basin, western Montana (Continued)

\begin{tabular}{|c|c|c|c|c|c|c|c|c|c|c|c|}
\hline $\begin{array}{c}\text { Site } \\
\text { number }\end{array}$ & Date & $\begin{array}{c}\text { Fluoride, } \\
\text { dissolved } \\
\text { (mg/L as } \\
\text { F) }\end{array}$ & $\begin{array}{c}\text { Silica, } \\
\text { dissolved } \\
(\mathrm{mg} / \mathrm{L} \text { as } \\
\left.\mathrm{SiO}_{2}\right)\end{array}$ & $\begin{array}{l}\text { Dissolved } \\
\text { solids, } \\
\text { calculated } \\
\text { (mg/L) }\end{array}$ & $\begin{array}{l}\text { Dissolved } \\
\text { solids } \\
\text { (ton/ } \\
\text { acre-ft) }\end{array}$ & $\begin{array}{c}\text { Dissolved } \\
\text { solids } \\
\text { (ton/day) }\end{array}$ & $\begin{array}{c}\text { Nitrite, } \\
\text { dissolved } \\
\text { (mg/L as } \\
\mathrm{N})\end{array}$ & $\begin{array}{l}\text { Nitrite plus } \\
\text { nitrate, } \\
\text { dissolved } \\
\text { (mg/L as } \\
\mathrm{N} \text { ) }\end{array}$ & $\begin{array}{c}\text { Ammonia, } \\
\text { dissolved } \\
(\mathrm{mg} / \mathrm{L} \text { as } \\
\mathrm{N})\end{array}$ & $\begin{array}{c}\text { Phosphorus, } \\
\text { orthophosphate, } \\
\text { dissolved } \\
\text { (mg/L as } \\
\text { P) }\end{array}$ & $\begin{array}{c}\text { Arsenic, } \\
\text { total. } \\
\text { recoverable } \\
(\mu \mathrm{g} / \mathrm{L} \text { as } \\
\text { As) }\end{array}$ \\
\hline \multirow[t]{8}{*}{ Sl } & $09-07-95$ & $<0.1$ & 10 & 175 & 0.24 & 1.33 & $<0 . i$ & $<0.05$ & $<0.02$ & $<0.01$ & $<1$ \\
\hline & $11-02-95$ & $<.1$ & 11 & 184 & .25 & 1.19 & $<.01$ & $<.05$ & $<.02$ & $<.01$ & $<1$ \\
\hline & $04-19-96$ & $<.1$ & 8.0 & 115 & .16 & 8.73 & .01 & $<.05$ & .02 & .01 & $<1$ \\
\hline & $06-20-96$ & $<.1$ & 8.4 & 181 & .25 & $6^{\prime} 4$ & $<.01$ & $<.05$ & .04 & $<.01$ & $<1$ \\
\hline & $08-21-96$ & $<.1$ & 9.6 & 143 & .19 & .92 & $<.01$ & $<.05$ & $<.02$ & $<.01$ & $<1$ \\
\hline & $10-24-96$ & $<.1$ & 10 & 194 & .26 & .63 & .01 & $<.05$ & .02 & $<.01$ & $<1$ \\
\hline & $04-14-97$ & $<.1$ & 9.0 & 256 & .35 & 2.07 & $<.01$ & .09 & $<.02$ & $<.01$ & $<1$ \\
\hline & $05-30-97$ & $<.1$ & 7.6 & 81 & .11 & 11.4 & $<.01$ & .05 & $<.02$ & $<.01$ & $<1$ \\
\hline \multirow[t]{8}{*}{ S2 } & $09-07-95$ & $<.1$ & 9.9 & 94 & .13 & .10 & $<.01$ & $<.05$ & $<.02$ & $<.01$ & $<1$ \\
\hline & $11-02-95$ & $<.1$ & 9.0 & 91 & .12 & .05 & $<.01$ & $<.05$ & $<.02$ & $<.01$ & $<1$ \\
\hline & $04-19-96$ & $<.1$ & 7.5 & 65 & .09 & 1.38 & .02 & $<.05$ & .02 & .01 & $<1$ \\
\hline & $06-20-96$ & $<.1$ & 8.7 & 78 & .11 & .32 & $<.01$ & $<.05$ & .03 & $<.01$ & $<1$ \\
\hline & $08-21-96$ & $<.1$ & 9.6 & 93 & .13 & .05 & $<.01$ & $<.05$ & $<.02$ & $<.01$ & $<1$ \\
\hline & $10-24-96$ & $<.1$ & 9.6 & 92 & .13 & .05 & .02 & $<.05$ & .02 & $<.01$ & 1 \\
\hline & $04-14-97$ & $<.1$ & 8.3 & 89 & .12 & .10 & $<.01$ & $<.05$ & $<.02$ & $<.01$ & $<1$ \\
\hline & $05-30-97$ & $<.1$ & 8.0 & 60 & .08 & 1.33 & $<.01$ & $<.05$ & $<.02$ & $<.01$ & $<1$ \\
\hline \multirow[t]{8}{*}{ S3 } & $09-07-95$ & $<.1$ & 12 & 134 & .18 & 2.17 & $<.01$ & $<.05$ & $<.02$ & $<.01$ & $<1$ \\
\hline & $11-02-95$ & $<.1$ & 12 & 131 & .18 & 2.87 & $<.01$ & $<.05$ & $<.02$ & $<.01$ & $<1$ \\
\hline & $04-19-96$ & $<.1$ & 8.6 & 95 & .13 & 25.4 & .02 & $<.05$ & .02 & $<.01$ & $<1$ \\
\hline & $06-20-96$ & $<.1$ & 9.6 & 139 & .19 & 9.76 & $<.01$ & $<.05$ & .03 & $<.01$ & $<1$ \\
\hline & $08-21-96$ & $<.1$ & 12 & 131 & .18 & 1.98 & $<.01$ & $<.05$ & $<.02$ & $<.01$ & $<1$ \\
\hline & $10-24-96$ & $<.1$ & 12 & 133 & .18 & 1.61 & .02 & $<.05$ & .02 & $<.01$ & $<1$ \\
\hline & $04-14-97$ & $<.1$ & 11 & 146 & .20 & 3.08 & $<.01$ & $<.05$ & $<.02$ & $<.01$ & $<1$ \\
\hline & $05-30-97$ & $<.1$ & 8.8 & 85 & .12 & 26.0 & $<.01$ & $<.05$ & $<.02$ & $<.01$ & $<1$ \\
\hline \multirow[t]{8}{*}{ S4 } & $09-07-95$ & $<.1$ & 11 & 132 & .18 & 3.92 & $<.01$ & $<.05$ & $<.02$ & $<.01$ & $<1$ \\
\hline & $11-02-95$ & $<.1$ & 11 & 137 & .19 & 2.25 & $<.01$ & $<.05$ & $<.02$ & $<.01$ & $<1$ \\
\hline & $04-19-96$ & $<.1$ & 8.9 & 96 & .13 & 26.5 & .02 & $<.05$ & .02 & .01 & $<1$ \\
\hline & $06-20-96$ & $<.1$ & 10 & 116 & .16 & 15.0 & $<.01$ & $<.05$ & .02 & $<.01$ & $<1$ \\
\hline & $08-21-96$ & $<.1$ & 11 & 134 & .18 & 4.27 & $<.01$ & $<.05$ & $<.02$ & .01 & $<1$ \\
\hline & $10-24-96$ & $<.1$ & 11 & 135 & .18 & 3.28 & .02 & $<.05$ & .02 & $<.01$ & $<1$ \\
\hline & $04-14-97$ & $<.1$ & 11 & 123 & .17 & 2.85 & $<.01$ & $<.05$ & $<.02$ & .02 & $<1$ \\
\hline & $05-30-97$ & $<.1$ & 9.3 & 93 & .13 & 33.0 & $<.01$ & $<.05$ & $<.02$ & $<.01$ & $<1$ \\
\hline \multirow[t]{8}{*}{ S5 } & $09-07-95$ & $<.1$ & 13 & 137 & .19 & 7.04 & $<.01$ & $<.05$ & $<.02$ & $<.01$ & $<1$ \\
\hline & $11-02-95$ & $<.1$ & 13 & 140 & .19 & 7.19 & $<.01$ & $<.05$ & $<.02$ & $<.01$ & $<1$ \\
\hline & $04-18-96$ & $<.1$ & 10 & 92 & .12 & 79.2 & .02 & $<.05$ & .02 & $<.01$ & $<1$ \\
\hline & $06-20-96$ & $<.1$ & 12 & 118 & .16 & 30.5 & $<.01$ & $<.05$ & .02 & $<.01$ & $<1$ \\
\hline & $08-21-96$ & $<.1$ & 13 & 136 & .18 & 9.16 & $<.01$ & $<.05$ & $<.02$ & $<.01$ & $<1$ \\
\hline & $10-24-96$ & $<.1$ & 13 & 142 & .19 & 6.90 & .01 & $<.05$ & .02 & $<.01$ & $<1$ \\
\hline & $04-14-97$ & $<.1$ & 12 & 134 & .18 & 10.1 & $<.01$ & $<.05$ & $<.02$ & $<.01$ & $<1$ \\
\hline & $05-30-97$ & $<.1$ & 10 & 88 & .12 & 75.2 & $<.01$ & $<.05$ & $<.02$ & $<.01$ & $<1$ \\
\hline
\end{tabular}


Table 2. Streamflow and water-quality data for the Blackfoot River basin, western Montana (Continued)

\begin{tabular}{|c|c|c|c|c|c|c|c|c|c|c|}
\hline $\begin{array}{c}\text { Site } \\
\text { number }\end{array}$ & Date & $\begin{array}{l}\text { Cadmium, } \\
\text { total } \\
\text { recoverable } \\
\text { ( } \mu \mathrm{g} / \mathrm{L} \text { as } \\
\text { Cd) }\end{array}$ & $\begin{array}{c}\text { Copper, } \\
\text { total } \\
\text { recoverable } \\
(\mu \mathrm{g} / \mathrm{L} \text { as } \\
\mathrm{Cu})\end{array}$ & $\begin{array}{c}\text { Iron, } \\
\text { total } \\
\text { recoverable } \\
(\mu \mathrm{g} / \mathrm{L} \text { as } \\
\mathrm{Fe})\end{array}$ & $\begin{array}{c}\text { Lead, } \\
\text { total } \\
\text { recoverable } \\
(\mu \mathrm{g} / \mathrm{L} \text { as } \\
\mathrm{Pb})\end{array}$ & $\begin{array}{c}\text { Manganese, } \\
\text { total } \\
\text { recoverable } \\
(\mu \mathrm{g} / \mathrm{L} \text { as } \\
\mathrm{Mn})\end{array}$ & $\begin{array}{c}\text { Zinc, } \\
\text { total } \\
\text { recoverable } \\
(\mu \mathrm{g} / \mathrm{L} \text { as } \\
\mathrm{Zn})\end{array}$ & $\begin{array}{c}\text { Sedi- } \\
\text { ment, } \\
\text { suspended } \\
(\mathbf{m g} / \mathbf{L})\end{array}$ & $\begin{array}{l}\text { Sediment, } \\
\text { discharge, } \\
\text { suspended } \\
(\text { ton } / d)\end{array}$ & $\begin{array}{l}\text { Sediment, } \\
\text { suspended, } \\
\text { (percent finer } \\
\text { than } 0.062 \mathrm{~mm} \text { ) }\end{array}$ \\
\hline \multirow[t]{8}{*}{ S1 } & $09-07-95$ & 5 & 8 & 60 & 8 & 450 & 1,600 & 3 & 0.02 & 82 \\
\hline & $11-02-95$ & 6 & 7 & 50 & 2 & 450 & 2,100 & 1 & .01 & 67 \\
\hline & $04-19-96$ & 5 & 34 & 110 & 12 & 350 & 1,300 & 1 & .08 & 62 \\
\hline & $06-20-96$ & 11 & 170 & 800 & 9 & 1,600 & 3,600 & 3 & .11 & 74 \\
\hline & $08-21-96$ & 3 & 9 & 80 & 3 & 220 & 850 & 1 & .01 & 38 \\
\hline & $10-24-96$ & 6 & 10 & 60 & 2 & 740 & 2,300 & 1 & $<.01$ & 57 \\
\hline & 04-14-97 & 6 & 15 & 80 & 3 & 730 & 2,500 & 1 & .01 & 57 \\
\hline & $05-30-97$ & 2 & 15 & 50 & 7 & 110 & 450 & 2 & .28 & 74 \\
\hline \multirow[t]{8}{*}{$\mathrm{S} 2$} & $09-07-95$ & $<1$ & $<1$ & $<10$ & $<1$ & 10 & $<10$ & 1 & $<.01$ & 67 \\
\hline & $11-02-95$ & $<1$ & $<1$ & 20 & $<1$ & $<10$ & $<10$ & 1 & $<.01$ & 75 \\
\hline & $04-19-96$ & $<1$ & 1 & 40 & $<1$ & $<10$ & $<10$ & 2 & .04 & 47 \\
\hline & $06-20-96$ & $<1$ & 1 & 20 & $<1$ & $<10$ & $<10$ & 1 & $<.01$ & 75 \\
\hline & $08-21-96$ & $<1$ & $<1$ & 20 & $<1$ & $<10$ & $<10$ & 1 & $<.01$ & 50 \\
\hline & $10-24-96$ & $<1$ & $<1$ & 20 & $<1$ & $<10$ & $<10$ & 1 & $<.01$ & 56 \\
\hline & $04-14-97$ & $<1$ & $<1$ & $<10$ & $<1$ & $<10$ & $<10$ & 1 & $<.01$ & 50 \\
\hline & $05-30-97$ & $<1$ & $<1$ & 30 & $<1$ & $<10$ & $<10$ & 1 & .02 & 62 \\
\hline \multirow[t]{8}{*}{ S3 } & $09-07-95$ & $<1$ & 1 & 140 & $<1$ & 80 & 90 & 1 & .02 & 80 \\
\hline & $11-02-95$ & $<1$ & $<1$ & 180 & $<1$ & 170 & 170 & 1 & .02 & 64 \\
\hline & $04-19-96$ & $<1$ & 3 & 120 & $<1$ & 30 & 170 & 1 & .27 & 50 \\
\hline & $06-20-96$ & $<1$ & 1 & 100 & $<1$ & 50 & 420 & 1 & .07 & 80 \\
\hline & $08-21-96$ & $<1$ & 1 & 180 & $<1$ & 80 & 90 & 2 & .03 & 60 \\
\hline & $10-24-96$ & $<1$ & 1 & 170 & $<1$ & 130 & 130 & 3 & .04 & 64 \\
\hline & $04-14-97$ & $<1$ & 1 & 170 & $<1$ & 80 & 290 & 1 & .02 & 78 \\
\hline & $05-30-97$ & $<1$ & 3 & 70 & $<1$ & 20 & 180 & 1 & .31 & 54 \\
\hline \multirow[t]{8}{*}{ S4 } & $09-07-95$ & $<1$ & $<1$ & 70 & $<1$ & 20 & $<10$ & 1 & .03 & 67 \\
\hline & $11-02-95$ & $<1$ & $<1$ & 70 & $<1$ & $<10$ & $<10$ & 1 & .02 & 71 \\
\hline & $04-19-96$ & $<1$ & 3 & 140 & $<1$ & $<10$ & $<10$ & 5 & 1.4 & 81 \\
\hline & $06-20-96$ & $<1$ & 1 & 120 & $<1$ & 10 & $<10$ & 3 & .39 & 89 \\
\hline & $08-21-96$ & $<1$ & $<1$ & 50 & $<1$ & $<10$ & $<10$ & 2 & .06 & 53 \\
\hline & $10-24-96$ & $<1$ & $<1$ & 60 & $<1$ & $<10$ & $<10$ & 1 & .02 & 70 \\
\hline & 04-14-97 & $<1$ & $<1$ & 90 & $<1$ & $<10$ & $<10$ & 1 & .02 & 75 \\
\hline & $05-30-97$ & $<1$ & 2 & 100 & $<1$ & 11 & $<10$ & 4 & 1.4 & 85 \\
\hline \multirow[t]{8}{*}{ S5 } & $09-07-95$ & $<1$ & $<1$ & 20 & $<1$ & $<10$ & $<10$ & 1 & .05 & 80 \\
\hline & $11-02-95$ & $<1$ & $<1$ & 70 & $<1$ & $<10$ & $<10$ & 2 & .10 & 74 \\
\hline & $04-18-96$ & $<1$ & 3 & 210 & $<1$ & 20 & 50 & 8 & 6.9 & 63 \\
\hline & $06-20-96$ & $<1$ & 1 & 100 & $<1$ & 10 & 60 & 3 & .78 & 88 \\
\hline & $08-21-96$ & $<1$ & $<1$ & 50 & $<1$ & $<10$ & $<10$ & 2 & .14 & 53 \\
\hline & $10-24-96$ & $<1$ & $<1$ & 30 & $<1$ & $<10$ & $<10$ & 3 & .15 & 61 \\
\hline & $04-14-97$ & $<1$ & 1 & 90 & $<1$ & $<10$ & 20 & 1 & .08 & 75 \\
\hline & $05-30-97$ & $<1$ & 3 & 120 & $<1$ & 15 & 60 & 5 & 4.3 & 69 \\
\hline
\end{tabular}


Table 2. Streamflow and water-quality data for the Blackfoot River basin, western Montana (Continued)

\begin{tabular}{|c|c|c|c|c|c|c|c|c|c|c|}
\hline $\begin{array}{c}\text { Site } \\
\text { number }\end{array}$ & Date & Time & $\begin{array}{c}\text { Streamflow, } \\
\text { instantaneous } \\
\left(\mathrm{ft}^{3} / \mathbf{s}\right)\end{array}$ & $\begin{array}{c}\text { Specific } \\
\text { conductance, } \\
\text { onsite } \\
(\mu \mathrm{S} / \mathrm{cm})\end{array}$ & $\begin{array}{c}\text { pH, } \\
\text { onsite } \\
\text { (standard } \\
\text { units) }\end{array}$ & $\begin{array}{c}\text { Tempera- } \\
\text { ture, } \\
\text { air } \\
\left({ }^{\circ} \mathrm{C}\right)\end{array}$ & $\begin{array}{c}\text { Tempera- } \\
\text { ture, } \\
\text { water } \\
\left({ }^{\circ} \mathrm{C}\right)\end{array}$ & $\begin{array}{c}\text { Oxygen, } \\
\text { dissolved, } \\
\text { onsite } \\
(\mathrm{mg} / \mathrm{L})\end{array}$ & $\begin{array}{c}\text { Oxygen, } \\
\text { dissolved, } \\
\text { onsite } \\
\text { (percent } \\
\text { saturation) }\end{array}$ & $\begin{array}{c}\text { Coliform, } \\
\text { fecal } \\
(\mathrm{col} / 100 \\
\mathrm{mL})\end{array}$ \\
\hline \multirow[t]{8}{*}{ S6 } & $09-05-95$ & 0830 & 70 & 260 & 8.2 & 12.0 & 9.5 & -. & -- & -- \\
\hline & $11-02-95$ & 1310 & 29 & 257 & 8.4 & -2.0 & 5.5 & -- & -- & -- \\
\hline & $04-18-96$ & 1510 & 238 & 230 & 8.3 & 4.0 & 4.0 & -- & -- & -- \\
\hline & $06-19-96$ & 1220 & 832 & 193 & 8.1 & 14.5 & 7.0 & -. & - & -- \\
\hline & $08-20-96$ & 1450 & 84 & 253 & 8.2 & 23.0 & 11.5 & -. & -- & -- \\
\hline & $10-23-96$ & 1515 & 34 & 261 & 7.1 & 4.0 & 5.0 & -- & -. & -- \\
\hline & $04-14-97$ & 1630 & 20 & 266 & 8.4 & 5.0 & 7.0 & -- & -- & -- \\
\hline & $05-30-97$ & 0900 & 1,050 & 197 & 8.2 & 13.0 & 5.5 & -- & -- & - \\
\hline \multirow[t]{8}{*}{ S7 } & $09-05-95$ & 1045 & 59 & 252 & 8.4 & 15.0 & 11.0 & 9.6 & 103 & K15 \\
\hline & $10-31-95$ & 0930 & 23 & 252 & 8.3 & -4.0 & 1.0 & 12.0 & 101 & 45 \\
\hline & $04-18-96$ & 1200 & 574 & 185 & 8.1 & 4.0 & 3.0 & 11.2 & 100 & -- \\
\hline & $06-19-96$ & 1430 & 893 & 193 & 8.3 & 9.0 & 9.0 & 10.6 & 109 & $<1$ \\
\hline & $08-20-96$ & 1300 & 89 & 248 & 8.4 & 22.5 & 11.0 & 9.4 & 101 & $\mathrm{~K} 2$ \\
\hline & $10-23-96$ & 1330 & 33 & 253 & 7.1 & 4.0 & 4.0 & 10.7 & 97 & 1 \\
\hline & $04-10-97$ & 1620 & 38 & 234 & 8.4 & -.5 & 2.0 & 12.7 & 108 & $<1$ \\
\hline & $05-27-97$ & 1500 & 1,300 & 189 & 8.4 & 16.0 & 9.0 & 9.9 & 101 & $\mathrm{~K} 1$ \\
\hline \multirow[t]{8}{*}{ S8 } & $09-05-95$ & 1300 & 110 & 310 & 8.4 & 23.0 & 14.0 & 9.3 & 102 & 22 \\
\hline & $10-31-95$ & 1200 & 93 & 322 & 8.2 & 0.0 & 3.5 & 11.8 & 105 & K1 \\
\hline & $04-18-96$ & 0900 & 802 & 224 & 8.0 & 2.0 & 3.0 & 9.6 & 85 & K5 \\
\hline & $06-19-96$ & 1700 & 1,170 & 213 & 8.2 & 9.0 & 11.0 & 9.3 & 99 & K3 \\
\hline & $08-20-96$ & 1030 & 169 & 301 & 8.3 & 10.5 & 9.5 & 8.9 & 92 & 20 \\
\hline & $10-23-96$ & 1110 & 98 & 324 & 7.8 & 3.5 & 6.0 & 10.1 & 96 & 7 \\
\hline & $04-10-97$ & 1400 & 81 & 324 & 8.3 & -1.0 & 5.0 & 9.8 & 90 & $<1$ \\
\hline & $05-29-97$ & 1520 & 1,470 & 214 & 8.3 & 19.0 & 11.5 & 9.4 & 101 & K3 \\
\hline \multirow[t]{8}{*}{ S9 } & 09-05-95 & 1520 & 160 & 297 & 8.4 & 21.0 & 17.0 & -- & -- & -- \\
\hline & $11-01-95$ & 0810 & 134 & 311 & 8.3 & -7.0 & 0.0 & -- & -- & -- \\
\hline & $04-17-96$ & 1630 & 956 & 228 & 8.3 & 7.0 & 6.5 & -- & -. & -- \\
\hline & $06-19-96$ & 0845 & 1,710 & 215 & 8.2 & 9.5 & 8.0 & -- & -- & -- \\
\hline & $08-20-96$ & 0730 & 226 & 293 & 8.4 & 4.0 & 12.0 & -- & -- & -- \\
\hline & $10-23-96$ & 0900 & 191 & 309 & 8.0 & 1.5 & 3.5 & -- & -- & - \\
\hline & $04-10-97$ & 1210 & 195 & 317 & 8.4 & 1.0 & 2.5 & -- & -- & -- \\
\hline & $05-29-97$ & 1220 & 2,040 & 221 & 8.2 & 22.5 & 10.5 & - & -- & - \\
\hline \multirow[t]{8}{*}{$\mathrm{S} 10$} & $09-06-95$ & 0930 & 30 & 429. & 8.3 & 13.0 & 16.0 & 6.7 & 79 & 180 \\
\hline & $10-31-95$ & 1445 & 24 & 450 & 8.6 & -2.0 & 1.0 & 12.8 & 105 & K7 \\
\hline & $04-17-96$ & 1400 & 330 & 278 & 8.7 & 8.0 & 6.5 & -- & -- & 24 \\
\hline & $06-18-96$ & 0930 & 150 & 310 & 8.2 & 7.0 & 14.0 & 9.5 & 108 & 350 \\
\hline & $08-19-96$ & 1100 & 28 & 383 & 8.5 & 22.0 & 16.0 & 11.8 & 139 & 98 \\
\hline & $10-22-96$ & 0930 & 33 & 426 & 8.4 & 3.0 & 2.5 & 11.4 & 98 & 61 \\
\hline & $04-09-97$ & 0940 & 117 & 457 & 8.2 & -1.0 & 0.0 & 13.2 & 105 & $\mathrm{~K} 150$ \\
\hline & $05-28-97$ & 1210 & 394 & 327 & 8.1 & 23.0 & 14.0 & - & - & 140 \\
\hline \multirow[t]{8}{*}{ S11 } & 09-06-95 & 1200 & 204 & 262 & 8.4 & 19.5 & 10.5 & - & -- & $\ldots$ \\
\hline & $11-01-95$ & 1010 & 156 & 269 & 8.4 & -2.5 & 3.5 & -- & -- & -- \\
\hline & $04-17-96$ & 1030 & 876 & 221 & 8.3 & 7.0 & 4.5 & - & -- & - \\
\hline & $06-18-96$ & 1230 & 2,530 & 147 & 8.4 & 9.0 & 6.5 & -- & -. & -- \\
\hline & $08-19-96$ & 1330 & 273 & 265 & 8.4 & 25.0 & 13.5 & -- & - & -- \\
\hline & $10-22-96$ & 1130 & 154 & 275 & 8.5 & 4.0 & 6.0 & -- & -- & -- \\
\hline & $04-10-97$ & 1030 & 155 & 281 & 8.4 & -1.0 & 2.5 & -- & -- & - \\
\hline & $05-28-97$ & 1430 & 2,470 & 174 & 8.2 & 24.0 & 8.5 & -- & -- & -- \\
\hline
\end{tabular}


Table 2. Streamflow and water-quality data for the Blackfoot River basin, western Montana (Continued)

\begin{tabular}{|c|c|c|c|c|c|c|c|c|c|c|c|}
\hline $\begin{array}{c}\text { Site } \\
\text { number }\end{array}$ & Date & $\begin{array}{l}\text { Streptococci, } \\
\text { fecal } \\
(\mathrm{col} / 100 \mathrm{~mL})\end{array}$ & $\begin{array}{c}\text { Hard- } \\
\text { ness, } \\
\text { total } \\
\left(\mathrm{mg}^{\prime} \mathrm{L} \text { as }\right. \\
\left.\mathrm{CaCO}_{3}\right) \\
\end{array}$ & $\begin{array}{l}\text { Calcium, } \\
\text { dissolved } \\
\text { (mg/L as } \\
\text { Ca) }\end{array}$ & $\begin{array}{l}\text { Magne- } \\
\text { sium, } \\
\text { dissolved } \\
\text { (mg/L as } \\
\mathrm{Mg} \text { ) }\end{array}$ & $\begin{array}{c}\text { Sodium, } \\
\text { dissolved } \\
\text { (mg/L as } \\
\mathrm{Na} \text { ) }\end{array}$ & $\begin{array}{l}\text { Sodium } \\
\text { adsorption } \\
\text { ratio }\end{array}$ & $\begin{array}{l}\text { Potassium, } \\
\text { dissolved } \\
\text { (mg/L as } \\
\mathrm{K} \text { ) }\end{array}$ & $\begin{array}{l}\text { Alkalinity, } \\
\text { laboratory } \\
\text { (mg/L as } \\
\left.\mathrm{CaCO}_{3}\right)\end{array}$ & $\begin{array}{c}\text { Sulfate, } \\
\text { dissolved } \\
(\mathrm{mg} / \mathrm{L} \text { as } \\
\left.\mathrm{SO}_{4}\right)\end{array}$ & $\begin{array}{c}\text { Chlo- } \\
\text { ride, } \\
\text { dissolved } \\
(\mathrm{mg} / \mathrm{L} \text { as } \\
\mathrm{Cl})\end{array}$ \\
\hline \multirow[t]{8}{*}{ S6 } & $09-05-95$ & -- & 130 & 34 & 12 & 1.1 & $<.1$ & .5 & 140 & 2.9 & .3 \\
\hline & $11-02-95$ & -- & 140 & 35 & 12 & 1.2 & $<.1$ & .5 & 139 & 3.0 & .3 \\
\hline & $04-18-96$ & -- & 120 & 31 & 11 & 1.1 & $<.1$ & .5 & 125 & 3.2 & .4 \\
\hline & $06-19-96$ & -- & 100 & 27 & 8.6 & .8 & $<.1$ & .4 & 99 & 1.6 & 2.0 \\
\hline & $08-20-96$ & -- & 130 & 34 & 11 & 1.1 & $<.1$ & .4 & 136 & 2.4 & 2.9 \\
\hline & $10-23-96$ & -- & 140 & 36 & 12 & 1.2 & $<.1$ & .5 & 142 & 3.0 & .2 \\
\hline & $04-14-97$ & -- & 140 & 37 & 12 & 1.2 & $<.1$ & .6 & 142 & 3.2 & .3 \\
\hline & $05-30-97$ & -- & 98 & 25 & 8.3 & .8 & $<.1$ & .4 & 103 & 1.7 & .2 \\
\hline \multirow[t]{8}{*}{ S7 } & $09-05-95$ & 24 & 130 & 32 & 12 & 1.5 & .1 & 6 & 133 & 5.0 & .4 \\
\hline & $10-31-95$ & $<1$ & 130 & 33 & 12 & 1.6 & .1 & .6 & 131 & 5.8 & .3 \\
\hline & $04-18-96$ & -- & 96 & 24 & 8.8 & 1.6 & .1 & .7 & 92 & 7.3 & .4 \\
\hline & $06-19-96$ & $\mathrm{~K} 1$ & 100 & 26 & 8.8 & 1.0 & $<.1$ & .4 & 101 & 3.3 & .2 \\
\hline & $08-20-96$ & 45 & 130 & 32 & 11 & 1.5 & .1 & .6 & 131 & 4.5 & .4 \\
\hline & $10-23-96$ & $<1$ & 130 & 33 & 12 & 1.7 & .1 & .6 & 134 & 5.8 & .2 \\
\hline & $04-10-97$ & $<1$ & 130 & 31 & 12 & 2.2 & .1 & .7 & 116 & 12 & .6 \\
\hline & $05-27-97$ & $\mathrm{~K} 1$ & 93 & 24 & 8.3 & 1.1 & .1 & .5 & 96 & 3.9 & .2 \\
\hline \multirow[t]{8}{*}{ S8 } & $09-05-95$ & K22 & 160 & 44 & 13 & 2.1 & .1 & .9 & 167 & 5.8 & .7 \\
\hline & $10-31-95$ & $\mathrm{~K} 3$ & 130 & 30 & 14 & 2.8 & .1 & .9 & -- & 5.5 & .6 \\
\hline & $04-18-96$ & $\mathrm{~K} 11$ & 120 & 31 & 9.7 & 1.8 & .1 & .8 & 114 & 7.1 & .5 \\
\hline & $06-19-96$ & K5 & 110 & 30 & 9.2 & 1.3 & .1 & .5 & 112 & 3.5 & .3 \\
\hline & $08-20-96$ & 37 & 150 & 42 & 12 & 1.9 & .1 & .8 & 160 & 5.1 & .5 \\
\hline & $10-23-96$ & 250 & 170 & 45 & 13 & 2.2 & .1 & .9 & 171 & 6.2 & .4 \\
\hline & $04-10-97$ & $\mathrm{~K} 3$ & 180 & 48 & 14 & 2.4 & .1 & .9 & 170 & 6.5 & 1.0 \\
\hline & $05-29-97$ & K11 & 110 & 28 & 8.7 & 1.4 & .1 & .6 & 110 & 4.1 & .3 \\
\hline \multirow[t]{8}{*}{ S9 } & $09-05-95$ & -- & 160 & 41 & 13 & 2.3 & .1 & .9 & 160 & 5.1 & .7 \\
\hline & $11-01-95$ & -- & 160 & 44 & 13 & 2.3 & .1 & .8 & 167 & 5.7 & .6 \\
\hline & $04-17-96$ & -- & 120 & 32 & 9.5 & 2.0 & .1 & .9 & 118 & 6.5 & .6 \\
\hline & $06-19-96$ & -- & 110 & 31 & 9.1 & 1.5 & .1 & .6 & 113 & 3.3 & .4 \\
\hline & $08-20-96$ & - & 150 & 41 & 12 & 2.0 & .1 & .8 & 157 & 5.0 & .6 \\
\hline & $10-23-96$ & -- & 160 & 43 & 13 & 2.3 & .1 & .9 & 165 & 5.5 & .4 \\
\hline & $04-10-97$ & -- & 170 & 46 & 14 & 2.8 & .1 & 1.0 & 166 & 6.3 & 1.0 \\
\hline & $05-29-97$ & - & 100 & 27 & 8.5 & 1.5 & .1 & .7 & 112 & 4.1 & .4 \\
\hline \multirow[t]{8}{*}{ S10 } & $09-06-95$ & 79 & 180 & 49 & 15 & 18 & .6 & 4.8 & 202 & 27 & 3.3 \\
\hline & $10-31-95$ & 22 & 200 & 56 & 15 & 18 & .6 & 4.1 & 209 & 34 & 3.4 \\
\hline & $04-17-96$ & 40 & 120 & 32 & 8.9 & 11 & .4 & 3.8 & 120 & 20 & 2.2 \\
\hline & $06-18-96$ & 200 & 140 & 39 & 11 & 11 & .4 & 3.1 & 149 & 14 & 1.5 \\
\hline & $08-19-96$ & 270 & 170 & 47 & 13 & 14 & .5 & 3.8 & 180 & 25 & 2.6 \\
\hline & $10-22-96$ & 650 & 190 & 53 & 14 & 15 & .5 & 3.9 & 196 & 33 & 3.3 \\
\hline & 04-09-97 & 730 & 200 & 53 & 17 & 23 & .7 & 4.3 & 174 & 58 & 4.8 \\
\hline & $05-28-97$ & 170 & 130 & 33 & 11 & 13 & .5 & 3.4 & 135 & 31 & 2.2 \\
\hline \multirow[t]{8}{*}{ S11 } & $09-06-95$ & -- & 140 & 31 & 14 & 1.4 & .1 & .5 & 140 & 3.9 & .3 \\
\hline & $11-01-95$ & -- & 140 & 32 & 14 & 1.4 & .1 & .5 & 143 & 4.2 & .3 \\
\hline & $04-17-96$ & -- & 120 & 30 & 10 & 1.1 & $<.1$ & .5 & 118 & $<.10$ & $<.1$ \\
\hline & $06-18-96$ & -- & 78 & 20 & 6.8 & .7 & $<.1$ & .4 & 78 & 1.5 & .1 \\
\hline & $08-19-96$ & -- & 140 & 32 & 14 & 1.3 & $<.1$ & .5 & 141 & 3.8 & .3 \\
\hline & $10-22-96$ & -- & 150 & 33 & 16 & 1.6 & .1 & .6 & 148 & 4.5 & .1 \\
\hline & $04-10-97$ & -- & 160 & 35 & 17 & 2.1 & .1 & .7 & 147 & 5.5 & .7 \\
\hline & $05-28-97$ & -- & 81 & 20 & 7.2 & .8 & $<.1$ & .4 & 89 & 2.0 & .2 \\
\hline
\end{tabular}


Table 2. Streamflow and water-quality data for the Blackfoot River basin, western Montana (Continued)

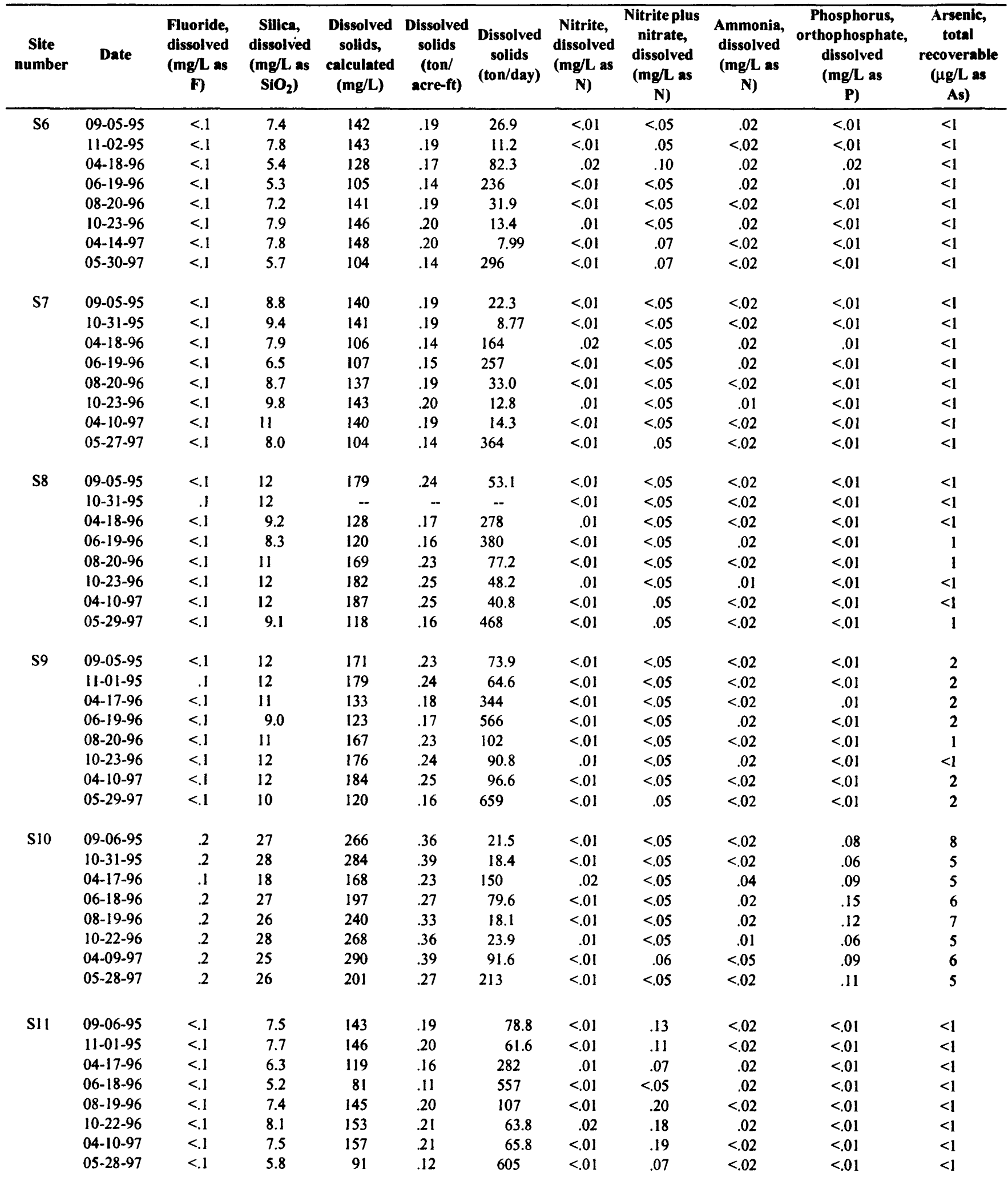


Table 2. Streamflow and water-quality data for the Blackfoot River basin, western Montana (Continued)

\begin{tabular}{|c|c|c|c|c|c|c|c|c|c|c|}
\hline $\begin{array}{c}\text { Site } \\
\text { number }\end{array}$ & Date & $\begin{array}{l}\text { Cadmium, } \\
\text { total } \\
\text { recoverable } \\
(\mu \mathrm{g} / \mathrm{L} \text { as } \\
\text { Cd) }\end{array}$ & $\begin{array}{l}\text { Copper, } \\
\text { total } \\
\text { recoverable } \\
(\mu \mathrm{g} / \mathrm{L} \text { as } \\
\mathrm{Cu})\end{array}$ & $\begin{array}{c}\text { Iron, } \\
\text { total } \\
\text { recoverable } \\
(\mu \mathrm{g} / \mathrm{L} \text { as } \\
\text { Fe })\end{array}$ & $\begin{array}{c}\text { Lead, } \\
\text { total } \\
\text { recoverable } \\
(\mu \mathrm{g} / \mathrm{L} \text { as } \\
\mathrm{Pb})\end{array}$ & $\begin{array}{c}\text { Manganese, } \\
\text { total } \\
\text { recoverable } \\
(\mu \mathrm{g} / \mathrm{L} \text { as } \\
\mathrm{Mn})\end{array}$ & $\begin{array}{c}\text { Zinc, } \\
\text { total } \\
\text { recoverable } \\
(\mu \mathrm{g} / \mathrm{L} \text { as } \\
\mathrm{Zn})\end{array}$ & $\begin{array}{l}\text { Sedi- } \\
\text { ment, } \\
\text { suspended } \\
(\mathbf{m g} / \mathbf{L})\end{array}$ & $\begin{array}{l}\text { Sediment, } \\
\text { discharge, } \\
\text { suspended } \\
\text { (ton/d) }\end{array}$ & $\begin{array}{l}\text { Sediment, } \\
\text { suspended, } \\
\text { (percent finer } \\
\text { than } 0.062 \mathrm{~mm} \text { ) }\end{array}$ \\
\hline \multirow[t]{8}{*}{ S6 } & $09-05-95$ & $<1$ & 1 & 450 & $<1$ & 20 & $<10$ & 38 & 7.2 & 98 \\
\hline & $11-02-95$ & $<1$ & $<1$ & $<10$ & $<1$ & 10 & $<10$ & 1 & .08 & 60 \\
\hline & $04-18-96$ & $<1$ & $<1$ & 70 & $<1$ & $<10$ & $<10$ & 5 & 3.2 & 84 \\
\hline & $06-19-96$ & $<1$ & 1 & 170 & $<1$ & 10 & $<10$ & 20 & 45 & 71 \\
\hline & $08-20-96$ & $<1$ & $<1$ & $<10$ & $<1$ & $<10$ & $<10$ & 2 & .45 & 53 \\
\hline & $10-23-96$ & $<1$ & $<1$ & $<10$ & $<1$ & $<10$ & $<10$ & 1 & .09 & 56 \\
\hline & $04-14-97$ & $<1$ & $<1$ & $<10$ & $<1$ & $<10$ & $<10$ & 2 & .11 & 88 \\
\hline & $05-30-97$ & $<1$ & 2 & 690 & 1 & 40 & $<10$ & 108 & 306 & 63 \\
\hline \multirow[t]{8}{*}{ S7 } & $09-05-95$ & $<1$ & 1 & 220 & $<1$ & 20 & $<10$ & 11 & 1.8 & 94 \\
\hline & $10-31-95$ & $<1$ & $<1$ & 20 & $<1$ & 10 & $<10$ & 1 & .06 & 67 \\
\hline & $04-18-96$ & $<1$ & 2 & 170 & $<1$ & 10 & 30 & 7 & 11 & 80 \\
\hline & $06-19-96$ & $<1$ & 2 & 180 & $<1$ & 10 & $<10$ & 23 & 55 & 65 \\
\hline & $08-20-96$ & $<1$ & $<1$ & $<10$ & $<1$ & $<10$ & $<10$ & 2 & .48 & 48 \\
\hline & $10-23-96$ & $<1$ & $<1$ & $<10$ & $<1$ & $<10$ & $<10$ & 1 & .09 & 50 \\
\hline & $04-10-97$ & $<1$ & $<1$ & 30 & $<1$ & $<10$ & $<10$ & 1 & .10 & 78 \\
\hline & $05-27-97$ & $<1$ & 3 & 420 & $<1$ & 27 & 20 & 50 & 176 & 76 \\
\hline \multirow[t]{8}{*}{ S8 } & $09-05-95$ & $<1$ & $<1$ & $<10$ & $<1$ & 10 & $<10$ & 7 & 2.1 & 68 \\
\hline & $10-31-95$ & $<1$ & $<1$ & 40 & $<1$ & 20 & $<10$ & 4 & 1.0 & 71 \\
\hline & $04-18-96$ & $<1$ & 4 & 200 & 1 & 20 & 20 & 20 & 32 & 73 \\
\hline & $06-19-96$ & $<1$ & 2 & 290 & $<1$ & 20 & $<10$ & 29 & 92 & 75 \\
\hline & $08-20-96$ & $<1$ & $<1$ & 50 & $<1$ & $<10$ & $<10$ & 2 & .91 & 88 \\
\hline & $10-23-96$ & $<1$ & $<1$ & 40 & $<1$ & $<10$ & $<10$ & 4 & 1.1 & 62 \\
\hline & $04-10-97$ & $<1$ & $<1$ & 60 & $<1$ & 10 & $<10$ & 9 & 2.0 & 65 \\
\hline & $05-29-97$ & $<1$ & 3 & 580 & $<1$ & 40 & $<10$ & 67 & 266 & 75 \\
\hline \multirow[t]{8}{*}{ S9 } & 09-05-95 & $<1$ & $<1$ & 30 & $<1$ & 20 & $<10$ & 5 & 2.2 & 74 \\
\hline & $11-01-95$ & $<1$ & $<1$ & 80 & $<1$ & 30 & $<10$ & 5 & 1.8 & 75 \\
\hline & $04-17-96$ & $<1$ & 5 & 760 & 2 & 40 & 10 & -- & -- & -- \\
\hline & $06-19-96$ & $<1$ & 3 & 630 & $<1$ & 50 & $<10$ & 65 & 300 & 82 \\
\hline & $08-20-96$ & $<1$ & $<1$ & 50 & $<1$ & $<10$ & $<10$ & 4 & 2.4 & 82 \\
\hline & $10-23-96$ & $<1$ & $<1$ & 60 & $<1$ & 10 & $<10$ & 5 & 2.6 & 80 \\
\hline & $04-10-97$ & $<1$ & $<1$ & 210 & $<1$ & 30 & $<10$ & 13 & 6.8 & 85 \\
\hline & $05-29-97$ & $<1$ & 4 & 950 & 2 & 60 & $<10$ & 92 & 507 & 85 \\
\hline \multirow[t]{8}{*}{ S10 } & $09-06-95$ & $<1$ & $<1$ & 70 & $<1$ & 20 & $<10$ & 3 & .24 & 97 \\
\hline & $10-31-95$ & $<1$ & $<1$ & 230 & $<1$ & 30 & $<10$ & 14 & .91 & 85 \\
\hline & $04-17-96$ & $<1$ & 3 & 800 & 1 & 70 & $<10$ & 40 & 38 & 92 \\
\hline & $06-18-96$ & $<1$ & 1 & 400 & $<1$ & 60 & $<10$ & 20 & 7.3 & 98 \\
\hline & $08-19-96$ & $<1$ & 1 & 190 & $<1$ & 30 & $<10$ & 6 & .45 & 89 \\
\hline & $10-22-96$ & $<1$ & $<1$ & 370 & $<1$ & 40 & $<10$ & 17 & 1.5 & 96 \\
\hline & 04-09-97 & $<1$ & 2 & 1,200 & $<1$ & 180 & $<10$ & 80 & 25 & 85 \\
\hline & $05-28-97$ & $<1$ & 2 & 440 & $<1$ & 56 & $<10$ & 33 & 35 & 83 \\
\hline \multirow[t]{8}{*}{ S11 } & $09-06-95$ & $<1$ & $<1$ & $<10$ & $<1$ & 10 & $<10$ & 1 & .55 & 78 \\
\hline & $11-01-95$ & $<1$ & $<1$ & 20 & $<1$ & $<10$ & $<10$ & 2 & .84 & 65 \\
\hline & $04-17-96$ & $<1$ & $<1$ & 80 & $<1$ & $<10$ & 10 & 7 & 17 & 83 \\
\hline & $06-18-96$ & $<1$ & 2 & 430 & 2 & 20 & $<10$ & 51 & 348 & 69 \\
\hline & $08-19-96$ & $<1$ & $<1$ & $<10$ & $<1$ & $<10$ & $<10$ & 1 & .74 & 60 \\
\hline & $10-22-96$ & $<1$ & $<1$ & $<10$ & $<1$ & $<10$ & $<10$ & 2 & .83 & 78 \\
\hline & $04-10-97$ & $<1$ & $<1$ & $<10$ & $<1$ & $<10$ & $<10$ & 1 & .42 & 60 \\
\hline & $05-28-97$ & $<1$ & 3 & 570 & $<1$ & 30 & $<10$ & 67 & 447 & 61 \\
\hline
\end{tabular}


Table 2. Streamflow and water-quality data for the Blackfoot River basin, western Montana (Continued)

\begin{tabular}{|c|c|c|c|c|c|c|c|c|c|c|}
\hline $\begin{array}{c}\text { Site } \\
\text { number }\end{array}$ & Date & Time & $\begin{array}{c}\text { Streamflow, } \\
\text { instantaneous } \\
\left(\mathbf{f t}^{3} / \mathbf{s}\right)\end{array}$ & $\begin{array}{c}\text { Specific } \\
\text { conductance, } \\
\text { onsite } \\
(\mu \mathrm{S} / \mathrm{cm})\end{array}$ & $\begin{array}{c}\text { pH, } \\
\text { onsite } \\
\text { (standard } \\
\text { units) }\end{array}$ & $\begin{array}{c}\text { Tempera- } \\
\text { ture, } \\
\text { air } \\
\left({ }^{\circ} \mathrm{C}\right)\end{array}$ & $\begin{array}{c}\text { Tempera- } \\
\text { ture, } \\
\text { water } \\
\left({ }^{\circ} \mathrm{C}\right)\end{array}$ & $\begin{array}{c}\text { Oxygen, } \\
\text { dissolved, } \\
\text { onsite } \\
(\mathrm{mg} / \mathrm{L})\end{array}$ & $\begin{array}{l}\text { Oxygen, } \\
\text { dissolved, } \\
\text { onsite } \\
\text { (percent } \\
\text { saturation) }\end{array}$ & $\begin{array}{c}\text { Coliform, } \\
\text { fecal } \\
\text { (col/100 } \\
\text { mL) }\end{array}$ \\
\hline \multirow[t]{8}{*}{$\mathrm{S} 12$} & $09-06-95$ & 1420 & 439 & 274 & 8.6 & 21.0 & 15.5 & 9.6 & 110 & 8 \\
\hline & $11-01-95$ & 1220 & 419 & 289 & 8.4 & -2.5 & 0.0 & 12.6 & 99 & -- \\
\hline & $04-16-96$ & 1330 & 2,530 & 216 & 8.3 & 10.5 & 5.0 & 11.8 & 109 & K2 \\
\hline & $06-18-96$ & 1545 & 5,870 & 170 & 8.0 & 7.0 & 6.5 & 11.7 & 109 & -- \\
\hline & $08-19-96$ & 1530 & 600 & 272 & 8.6 & 26.0 & 16.0 & 11.4 & 133 & K4 \\
\hline & $10-22-96$ & 1320 & 460 & 299 & 8.5 & 5.0 & 5.0 & 12.6 & 114 & K4 \\
\hline & $04-09-97$ & 1420 & 697 & 336 & 8.7 & 3.5 & 4.5 & 14.2 & 126 & $<1$ \\
\hline & $05-29-97$ & 0830 & 6,060 & 171 & 8.1 & 17.0 & 8.5 & 10.1 & 100 & 30 \\
\hline \multirow[t]{8}{*}{$\mathrm{S} 13$} & 09-06-95 & 1645 & 42 & 142 & 8.8 & 25.5 & 21.5 & -- & -- & -- \\
\hline & $11-01-95$ & 1500 & 89 & 141 & 8.5 & -1.0 & 5.5 & -- & -- & -- \\
\hline & $04-17-96$ & 0815 & 1,010 & 103 & 8.5 & 3.5 & 3.5 & -- & - & - \\
\hline & $06-18-96$ & 1800 & 1,110 & 104 & 7.9 & 8.0 & 16.0 & -- & -- & -- \\
\hline & $08-19-96$ & 1800 & 103 & 137 & 8.7 & 23.0 & 20.5 & -- & -- & -- \\
\hline & $10-22-96$ & 1530 & 54 & 150 & 8.7 & 7.0 & 8.0 & -- & -- & -- \\
\hline & $04-09-97$ & 1645 & 390 & 141 & 8.3 & 2.0 & 4.5 & -- & -- & -- \\
\hline & $05-28-97$ & 1800 & 2,320 & 98 & 7.8 & 24.0 & 11.5 & -- & -- & -- \\
\hline
\end{tabular}


Table 2. Streamflow and water-quality data for the Blackfoot River basin, western Montana (Continued)

\begin{tabular}{|c|c|c|c|c|c|c|c|c|c|c|c|}
\hline $\begin{array}{c}\text { Site } \\
\text { number }\end{array}$ & Date & $\begin{array}{l}\text { Streptococci, } \\
\text { fecal } \\
(\mathrm{col} / 100 \mathrm{~mL})\end{array}$ & $\begin{array}{c}\text { Hard- } \\
\text { ness, } \\
\text { total } \\
\left(\mathrm{mg}^{\prime} / \mathrm{L} \text { as }\right. \\
\left.\mathrm{CaCO}_{3}\right)\end{array}$ & $\begin{array}{l}\text { Calcium, } \\
\text { dissolved } \\
\text { (mg/L as } \\
\text { Ca) }\end{array}$ & $\begin{array}{l}\text { Magne- } \\
\text { sium, } \\
\text { dissolved } \\
\text { (mg/L as } \\
\text { Mg) }\end{array}$ & $\begin{array}{c}\text { Sodium, } \\
\text { dissolved } \\
\text { (mg/L as } \\
\mathrm{Na})\end{array}$ & $\begin{array}{l}\text { Sodium } \\
\text { adsorption } \\
\text { ratio }\end{array}$ & $\begin{array}{l}\text { Potassium, } \\
\text { dissolved } \\
\text { (mg/L as } \\
\text { K) }\end{array}$ & $\begin{array}{l}\text { Alkalinity, } \\
\text { laboratory } \\
\text { (mg/L as } \\
\left.\mathrm{CaCO}_{3}\right)\end{array}$ & $\begin{array}{c}\text { Sulfate, } \\
\text { dissolved } \\
\text { (mg/L as } \\
\left.\mathrm{SO}_{4}\right)\end{array}$ & $\begin{array}{c}\text { Chlo- } \\
\text { ride, } \\
\text { dissolved } \\
\text { (mg/L as } \\
\mathrm{Cl})\end{array}$ \\
\hline \multirow[t]{6}{*}{ S12 } & $09-06-95$ & 9 & 140 & 34 & 13 & 2.8 & .1 & .9 & 146 & 5.7 & .6 \\
\hline & $11-01-95$ & -- & 150 & 37 & 14 & 3.0 & .1 & .9 & 155 & 6.5 & .6 \\
\hline & $04-16-96$ & K8 & 100 & 26 & 8.7 & 2.6 & .1 & 1.2 & 103 & 6.7 & .6 \\
\hline & $10-22-96$ & -- & 150 & 37 & 13 & 3.6 & .1 & 1.1 & 157 & 7.5 & .7 \\
\hline & $04-09-97$ & - & 170 & 43 & 16 & 7.5 & .2 & 1.8 & 157 & 20 & 2.2 \\
\hline & $05-29-97$ & 30 & 80 & 20 & 6.9 & 1.7 & .1 & .7 & 85 & 4.1 & .3 \\
\hline \multirow[t]{4}{*}{$\mathrm{S} 13$} & $09-06-95$ & -- & 72 & 19 & 5.9 & 1.4 & .1 & .5 & 75 & 1.4 & .5 \\
\hline & $11-01-95$ & -- & 72 & 19 & 5.9 & 1.5 & .1 & .5 & 74 & 1.4 & .4 \\
\hline & 04-09-97 & -- & 75 & 20 & 6.3 & 1.7 & .1 & .6 & 72 & 1.8 & .6 \\
\hline & $05-28-97$ & -- & 46 & 12 & 3.8 & 1.0 & .1 & .5 & 49 & 1.2 & .4 \\
\hline
\end{tabular}


Table 2. Streamflow and water-quality data for the Blackfoot River basin, western Montana (Continued)

\begin{tabular}{|c|c|c|c|c|c|c|c|c|c|c|c|}
\hline $\begin{array}{c}\text { Site } \\
\text { number }\end{array}$ & Date & $\begin{array}{c}\text { Fluoride, } \\
\text { dissolved } \\
\text { (mg/L as } \\
\text { F) }\end{array}$ & $\begin{array}{l}\text { Silica, } \\
\text { dissolved } \\
(\mathbf{m g} / \mathrm{L} \text { as } \\
\left.\mathrm{SiO}_{2}\right)\end{array}$ & $\begin{array}{l}\text { Dissolved } \\
\text { solids, } \\
\text { calculated } \\
\text { (mg/L) }\end{array}$ & $\begin{array}{l}\text { Dissolved } \\
\text { solids } \\
\text { (ton/ } \\
\text { acre-ft) }\end{array}$ & $\begin{array}{l}\text { Dissolved } \\
\text { solids } \\
\text { (ton/day) }\end{array}$ & $\begin{array}{l}\text { Nitrite, } \\
\text { dissolved } \\
\text { (mg/L as } \\
\text { N) }\end{array}$ & $\begin{array}{l}\text { Nitrite plus } \\
\text { nitrate, } \\
\text { dissolved } \\
\text { (mg/L as } \\
\mathrm{N} \text { ) }\end{array}$ & $\begin{array}{c}\text { Ammonia, } \\
\text { dissolved } \\
\text { (mg/L as } \\
\mathrm{N})\end{array}$ & $\begin{array}{l}\text { Phosphorus, } \\
\text { orthophosphate, } \\
\text { dissolved } \\
\text { (mg/L as } \\
\text { P) }\end{array}$ & $\begin{array}{c}\text { Arsenic, } \\
\text { total } \\
\text { recoverable } \\
(\mu \mathrm{g} / \mathrm{L} \text { as } \\
\text { As })\end{array}$ \\
\hline \multirow[t]{8}{*}{$\mathrm{S} 12$} & $09-06-95$ & $<.1$ & 9.7 & 154 & .21 & 183 & $<.01$ & $<.05$ & $<.02$ & $<.01$ & 1 \\
\hline & $11-01-95$ & $<.1$ & 11 & 166 & .23 & 188 & $<.01$ & $<.05$ & $<.02$ & $<.01$ & 1 \\
\hline & $04-16-96$ & $<.1$ & 10 & 118 & .16 & 805 & .01 & .05 & .02 & .02 & 2 \\
\hline & $06-18-96$ & $<.1$ & 7.3 & 91 & .12 & 1,450 & $<.01$ & $<.05$ & .03 & $<.01$ & 1 \\
\hline & $08-19-96$ & $<.1$ & 9.2 & 151 & .21 & 245 & $<.01$ & .07 & .02 & $<.01$ & $<1$ \\
\hline & $10-22-96$ & $<.1$ & 11 & 168 & .23 & 209 & .01 & $<.02$ & .04 & $<.01$ & 1 \\
\hline & $04-09-97$ & .1 & 13 & 198 & .27 & 373 & $<.01$ & $<.05$ & $<.02$ & .01 & 2 \\
\hline & $05-29-97$ & $<.1$ & 8.2 & 94 & .13 & 1,540 & $<.01$ & .06 & $<.02$ & .02 & 1 \\
\hline \multirow[t]{8}{*}{$\mathrm{S} 13$} & $09-06-95$ & $<.1$ & 6.5 & 80 & .11 & 9.1 & $<.01$ & $<.05$ & $<.02$ & $<.01$ & $<1$ \\
\hline & $11-01-95$ & $<.1$ & 7.4 & 80 & .11 & 19.3 & $<.01$ & $<.05$ & $<.02$ & $<.01$ & $<1$ \\
\hline & $04-17-96$ & $<.1$ & 8.9 & 71 & .10 & 193 & .02 & $<.05$ & .02 & $<.01$ & $<1$ \\
\hline & $06-18-96$ & $<.1$ & 6.5 & 59 & .08 & 175 & $<.01$ & $<.05$ & .02 & $<.01$ & $<1$ \\
\hline & $08-19-96$ & $<.1$ & 5.9 & 76 & .10 & 21.1 & $<.01$ & $<.05$ & $<.02$ & $<.01$ & $<1$ \\
\hline & $10-22-96$ & $<.1$ & 7.8 & 84 & .11 & 12.3 & $<.01$ & $<.05$ & .01 & $<.01$ & $<1$ \\
\hline & $04-09-97$ & $<.1$ & 8.6 & 82 & .11 & 86.5 & $<.01$ & $<.05$ & $<.02$ & $<.01$ & $<1$ \\
\hline & $05-28-97$ & $<.1$ & 8.0 & 56 & .08 & 354 & $<.01$ & $<.05$ & $<.02$ & $<.01$ & $<1$ \\
\hline
\end{tabular}


Table 2. Streamflow and water-quality data for the Blackfoot River basin, western Montana (Continued)

\begin{tabular}{|c|c|c|c|c|c|c|c|c|c|c|}
\hline $\begin{array}{c}\text { Site } \\
\text { number }\end{array}$ & Date & $\begin{array}{l}\text { Cadmium, } \\
\text { total } \\
\text { recoverable } \\
\text { ( } \mu \mathrm{g} / \mathrm{L} \text { as } \\
\text { Cd) }\end{array}$ & $\begin{array}{l}\text { Copper, } \\
\text { total } \\
\text { recoverable } \\
\text { ( } \mu \mathrm{g} / \mathrm{L} \text { as } \\
\mathrm{Cu})\end{array}$ & $\begin{array}{c}\text { Iron, } \\
\text { total } \\
\text { recoverable } \\
(\mu \mathrm{g} / \mathrm{L} \text { as } \\
\mathrm{Fe})\end{array}$ & $\begin{array}{c}\text { Lead, } \\
\text { total } \\
\text { recoverable } \\
(\mu \mathrm{g} / \mathrm{L} \text { as } \\
\mathrm{Pb})\end{array}$ & $\begin{array}{c}\text { Manganese, } \\
\text { total } \\
\text { recoverable } \\
(\mu \mathrm{g} / \mathrm{L} \text { as } \\
\mathrm{Mn})\end{array}$ & $\begin{array}{c}\text { Zinc, } \\
\text { total } \\
\text { recoverable } \\
(\mu \mathrm{g} / \mathrm{L} \text { as } \\
\mathrm{Zn})\end{array}$ & $\begin{array}{c}\text { Sedi- } \\
\text { ment, } \\
\text { suspended } \\
(\mathbf{m g} / \mathbf{L})\end{array}$ & $\begin{array}{l}\text { Sediment, } \\
\text { discharge, } \\
\text { suspended } \\
\text { (ton/d) }\end{array}$ & $\begin{array}{l}\text { Sediment, } \\
\text { suspended, } \\
\text { (percent finer } \\
\text { than } 0.062 \mathrm{~mm} \text { ) }\end{array}$ \\
\hline \multirow[t]{6}{*}{$\$ 12$} & $09-06-95$ & $<1$ & $<1$ & 20 & $<1$ & $<10$ & $<10$ & 2 & 2.4 & 86 \\
\hline & $11-01-95$ & $<1$ & $<1$ & 40 & $<1$ & 10 & $<10$ & 4 & 4.5 & 81 \\
\hline & $04-16-96$ & $<1$ & 4 & 430 & 2 & 30 & $<10$ & 40 & 280 & 75 \\
\hline & $10-22-96$ & $<1$ & $<1$ & 40 & $<1$ & $<10$ & $<10$ & 3 & 3.7 & 77 \\
\hline & $04-09-97$ & $<1$ & $<1$ & 260 & $<1$ & 30 & $<10$ & 13 & 24 & 98 \\
\hline & $05-29-97$ & $<1$ & 3 & 610 & $<1$ & 40 & $<10$ & 67 & 1,100 & 75 \\
\hline \multirow[t]{4}{*}{ S13 } & $09-06-95$ & $<1$ & $<1$ & $<10$ & $<1$ & 10 & $<10$ & 1 & .11 & 88 \\
\hline & $11-01-95$ & $<1$ & $<1$ & 40 & $<1$ & 20 & $<10$ & 3 & .72 & 57 \\
\hline & $04-09-97$ & $<1$ & $<1$ & 70 & $<1$ & $<10$ & $<10$ & 2 & 2.1 & 79 \\
\hline & $05-28-97$ & $<1$ & 1 & 100 & $<1$ & 11 & $<10$ & 4 & 25 & 90 \\
\hline
\end{tabular}


Table 3. Water-quality data for replicate samples from sites in the Blackfoot River basin, western Montana

[Site numbers shown in figure 1 and described in text. Abbreviations: $\mu \mathrm{S} / \mathrm{cm}$, microseimens per centimeter at $25^{\circ} \mathrm{C} ; \mathrm{mg} / \mathrm{L} \mathrm{milligrams} \mathrm{per} \mathrm{liter;} \mathrm{lab,} \mathrm{laboratory;}$ ton/acre-ft, tons per acre-foot; $\mu \mathrm{g} / \mathrm{L}$, micrograms per liter; mm, millimeter. Symbols: <, less than minimum reporting level; --, no data]

\begin{tabular}{|c|c|c|c|c|c|c|c|c|c|c|c|}
\hline $\begin{array}{c}\text { Site } \\
\text { number }\end{array}$ & Date & Time & $\begin{array}{c}\text { Specific } \\
\text { conduct- } \\
\text { ance, } \\
\text { onsite } \\
(\mu \mathrm{S} / \mathrm{cm})\end{array}$ & $\begin{array}{c}\text { pH, } \\
\text { onsite } \\
\text { (stan- } \\
\text { dard units) }\end{array}$ & $\begin{array}{c}\text { Hardness, } \\
\text { total } \\
\text { (mg/L } \\
\text { as } \\
\left.\mathrm{CaCO}_{3}\right)\end{array}$ & $\begin{array}{c}\text { Calcium, } \\
\text { dis- } \\
\text { solved } \\
\text { (mg/L } \\
\text { as Ca) }\end{array}$ & $\begin{array}{c}\text { Mag- } \\
\text { nesium, } \\
\text { dissolved } \\
\text { (mg/L as } \\
\mathrm{Mg} \text { ) }\end{array}$ & $\begin{array}{l}\text { Sodium, } \\
\text { dis- } \\
\text { solved } \\
\text { (mg/L as } \\
\text { Na) }\end{array}$ & $\begin{array}{c}\text { Sodium } \\
\text { adsorp- } \\
\text { tion } \\
\text { ratio }\end{array}$ & $\begin{array}{l}\text { Potassium, } \\
\text { dis- } \\
\text { solved } \\
\text { (mg/L as } \\
\text { K) }\end{array}$ & $\begin{array}{c}\text { Alka- } \\
\text { linity, } \\
\text { lab } \\
\text { (mg/L as } \\
\mathrm{CaCO}_{3} \text { ) }\end{array}$ \\
\hline \multirow[t]{2}{*}{ S2 } & $06-20-96$ & 1330 & 137 & 8.1 & 67 & 16 & 6.5 & 1.8 & 0.1 & 0.3 & 69 \\
\hline & & 1335 & 137 & 8.1 & 69 & 17 & 6.5 & 1.8 & .1 & .3 & 69 \\
\hline \multirow[t]{2}{*}{ S5 } & $04-14-97$ & 1520 & 232 & 8.4 & 120 & 27 & 12 & 2.3 & .1 & .9 & 103 \\
\hline & & 1525 & 232 & 8.4 & 110 & 27 & 12 & 2.3 & .1 & .8 & 103 \\
\hline \multirow[t]{2}{*}{ S7 } & $04-18-96$ & 1200 & 185 & 8.1 & 96 & 24 & 8.8 & 1.6 & .1 & .7 & 92 \\
\hline & & 1205 & 185 & 8.1 & 96 & 24 & 8.7 & 1.6 & .1 & .7 & 92 \\
\hline \multirow[t]{4}{*}{ S9 } & $08-20-96$ & 0730 & 293 & 8.4 & 150 & 41 & 12 & 2.0 & .1 & .8 & 157 \\
\hline & & 0735 & 293 & 8.4 & 150 & 40 & 12 & 2.0 & .1 & .9 & 157 \\
\hline & $10-23-96$ & 0900 & 309 & 8.0 & 160 & 43 & 13 & 2.3 & .1 & .9 & 165 \\
\hline & & 0905 & 309 & 8.0 & 140 & 42 & 13 & 2.4 & .1 & .9 & 165 \\
\hline \multirow[t]{2}{*}{$\mathrm{S} 12$} & $11-01-95$ & 1220 & 289 & 8.4 & 150 & 37 & 14 & 3.0 & .1 & .9 & 155 \\
\hline & & 1225 & 289 & 8.4 & 150 & 37 & 14 & 3.1 & .1 & .9 & 154 \\
\hline \multirow[t]{2}{*}{$\mathrm{S} 13$} & $05-28-97$ & 1800 & 98 & 7.8 & 46 & 12 & 3.8 & 1.0 & .1 & .5 & 49 \\
\hline & & 1805 & 98 & 7.8 & 46 & 12 & 3.7 & 1.0 & .1 & .5 & 49 \\
\hline
\end{tabular}

\begin{tabular}{|c|c|c|c|c|c|c|c|c|c|c|c|}
\hline $\begin{array}{c}\text { Site } \\
\text { number }\end{array}$ & Date & $\begin{array}{c}\text { Sulfate, } \\
\text { dissolved } \\
\text { (mg/L } \\
\text { as } \\
\mathrm{SO}_{4} \text { ) }\end{array}$ & $\begin{array}{l}\text { Chloride, } \\
\text { dissolved } \\
\text { (mg/L as } \\
\text { Cl) }\end{array}$ & $\begin{array}{c}\text { Fluoride, } \\
\text { dissolved } \\
\text { (mg/L as F) }\end{array}$ & $\begin{array}{c}\text { Silica, } \\
\text { dissolved } \\
(\mathrm{mg} / \mathrm{L} \text { as } \\
\left.\mathrm{SiO}_{2}\right)\end{array}$ & $\begin{array}{l}\text { Dissolved } \\
\text { solids, } \\
\text { calculated } \\
(\mathrm{mg} / \mathrm{L})\end{array}$ & $\begin{array}{l}\text { Dissolved } \\
\text { solids } \\
\text { (tons/ } \\
\text { acre-ft) }\end{array}$ & $\begin{array}{c}\text { Nitrite, } \\
\text { dissolved } \\
\text { (mg/L as } \mathbf{N})\end{array}$ & $\begin{array}{l}\text { Nitrite plus } \\
\text { nitrate, } \\
\text { dissolved } \\
\text { (mg/L as } \mathbf{N} \text { ) }\end{array}$ & $\begin{array}{c}\text { Ammonia, } \\
\text { dissolved } \\
\text { (mg/L as } \mathbf{N})\end{array}$ & $\begin{array}{c}\text { Phosphorus, } \\
\text { orthophos- } \\
\text { phate, } \\
\text { dissolved } \\
\text { (mg/L as P) }\end{array}$ \\
\hline \multirow[t]{2}{*}{ S2 } & $06-20-96$ & 3.1 & 0.5 & $<0.1$ & 8.7 & 78 & 0.11 & $<0.01$ & $<0.05$ & 0.03 & $<0.01$ \\
\hline & & 3.0 & .5 & $<.1$ & 8.6 & 79 & .11 & $<.01$ & $<.05$ & .02 & $<.01$ \\
\hline \multirow[t]{2}{*}{ S3 } & $04-14-97$ & 17 & .8 & $<.1$ & 12 & 134 & .18 & $<.01$ & $<.05$ & $<.02$ & $<.01$ \\
\hline & & 17 & .7 & $<.1$ & 12 & 133 & .18 & $<.01$ & $<.05$ & $<.02$ & $<.01$ \\
\hline \multirow[t]{2}{*}{ S7 } & $04-18-96$ & 7.3 & .4 & $<.1$ & 7.9 & 106 & .14 & .02 & $<.05$ & .02 & .01 \\
\hline & & 7.3 & .4 & $<.1$ & 9.5 & 107 & .15 & .02 & $<.05$ & .02 & .01 \\
\hline \multirow[t]{4}{*}{ S9 } & $08-20-96$ & 5.0 & 6 & $<.1$ & 11 & 167 & .23 & $<.01$ & $<.05$ & $<.02$ & $<.01$ \\
\hline & & 4.7 & .7 & $<.1$ & 11 & 165 & .22 & $<.01$ & $<.05$ & .02 & .01 \\
\hline & $10-23-96$ & 5.5 & .4 & $<.1$ & 12 & 176 & .24 & .01 & $<.05$ & .02 & $<.01$ \\
\hline & & 5.6 & .4 & $<.1$ & 12 & 175 & .24 & .02 & $<.05$ & .01 & $<.01$ \\
\hline \multirow[t]{2}{*}{$\mathrm{S} 12$} & $11-01-95$ & 6.5 & .6 & $<.1$ & 11 & 166 & .23 & $<.01$ & $<.05$ & $<.02$ & $<.01$ \\
\hline & & 6.6 & .6 & $<.1$ & 11 & 166 & .23 & $<.01$ & $<.05$ & $<.02$ & $<.01$ \\
\hline \multirow[t]{2}{*}{$\mathrm{S} 13$} & $05-28-97$ & 1.2 & .4 & $<.1$ & 8.0 & 56 & .08 & $<.01$ & $<.05$ & $<.02$ & $<.01$ \\
\hline & & 1.2 & .6 & $<.1$ & 8.0 & 57 & .08 & - & - & - & - \\
\hline
\end{tabular}

\begin{tabular}{|c|c|c|c|c|c|c|c|c|c|c|}
\hline $\begin{array}{c}\text { Site } \\
\text { number }\end{array}$ & Date & $\begin{array}{l}\text { Arsenic, } \\
\text { total recov- } \\
\text { erable } \\
(\mu \mathrm{g} / \mathrm{L} \text { as } \\
\text { As) }\end{array}$ & $\begin{array}{l}\text { Cadmium, } \\
\text { total } \\
\text { recov- } \\
\text { erable } \\
\text { ( } \mu \mathrm{g} / \mathrm{L} \text { as } \\
\text { Cd) }\end{array}$ & 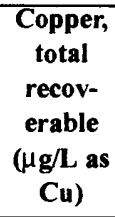 & $\begin{array}{l}\text { Iron, } \\
\text { total } \\
\text { recov- } \\
\text { erable } \\
(\mu \mathrm{g} / \mathrm{L} \text { as } \\
\mathrm{Fe})\end{array}$ & $\begin{array}{c}\text { Lead, } \\
\text { total recov- } \\
\text { erable } \\
(\mu \mathrm{g} / \mathrm{L} \text { as } \\
\text { Pb) }\end{array}$ & $\begin{array}{c}\text { Manganese, } \\
\text { total recov- } \\
\text { erable } \\
(\mu \mathrm{g} / \mathrm{L} \text { as } \\
\mathrm{Mn})\end{array}$ & $\begin{array}{c}\text { Zinc, } \\
\text { total recov- } \\
\text { erable } \\
(\mu \mathrm{g} / \mathrm{L} \text { as } \\
\left.\mathrm{Zn}_{\mathrm{n}}\right)\end{array}$ & $\begin{array}{c}\text { Sedi- } \\
\text { ment, } \\
\text { suspended } \\
\text { (mg/L) }\end{array}$ & $\begin{array}{r}\text { Sediment } \\
\text { suspended, } \\
\text { (percent } \\
\text { finer than } \\
0.062 \mathrm{~mm} \text { ) }\end{array}$ \\
\hline \multirow[t]{2}{*}{$\mathrm{S} 2$} & $06-20-96$ & $<1$ & $<1$ & 1 & 20 & $<1$ & $<10$ & $<10$ & 1 & 75 \\
\hline & & $<1$ & $<1$ & $<1$ & 20 & $<1$ & $<10$ & $<10$ & - & -- \\
\hline \multirow[t]{2}{*}{ S3 } & $04-14-97$ & $<1$ & $<1$ & 1 & 90 & $<1$ & $<10$ & 20 & 1 & 75 \\
\hline & & $<1$ & $<1$ & $<1$ & 90 & $<1$ & $<10$ & 20 & - & $\ldots$ \\
\hline \multirow[t]{2}{*}{ S7 } & $04-18-96$ & $<1$ & $<1$ & 2 & 170 & $<1$ & 10 & 30 & 7 & 80 \\
\hline & & $<1$ & $<1$ & 2 & 170 & $<1$ & 10 & 30 & 7 & 81 \\
\hline \multirow[t]{4}{*}{ S9 } & $08-20-96$ & 2 & $<1$ & $<1$ & 50 & $<1$ & $<10$ & $<10$ & 4 & 82 \\
\hline & & $<1$ & $<1$ & $<1$ & 60 & $<1$ & $<10$ & $<10$ & - & -- \\
\hline & $10-23-96$ & $<1$ & $<$ I & $<1$ & 60 & $<1$ & 10 & $<10$ & 5 & 80 \\
\hline & & $<1$ & $<1$ & $<1$ & 60 & $<1$ & 10 & $<10$ & 6 & 76 \\
\hline \multirow[t]{2}{*}{$\mathrm{S} 12$} & $11-01-95$ & 1 & $<1$ & $<1$ & 40 & $<1$ & 10 & $<10$ & 4 & 81 \\
\hline & & 2 & $<1$ & $<1$ & 40 & $<1$ & 10 & $<10$ & - & - \\
\hline \multirow[t]{2}{*}{ S13 } & $05-28-97$ & $<1$ & $<1$ & 1 & 100 & $<1$ & 11 & $<10$ & 4 & 90 \\
\hline & & 2 & $<1$ & $<1$ & 110 & $<1$ & 12 & $<10$ & - & - \\
\hline
\end{tabular}


Table 4. Water-quality data for field-blank samples from sites in the Blackfoot River basin, western Montana

[Site numbers shown in figure 1 and described in text. Abbreviations: $\mu \mathrm{S} / \mathrm{cm}$, microseimens per centimeter at $25^{\circ} \mathrm{C} ; \mathrm{mg} / \mathrm{L}$ milligrams per liter; lab, laboratory; ton/acre-ft, tons per acre-foot; $\mu \mathrm{g} / \mathrm{L}$, micrograms per liter; mm, millimeter. Symbols:

$<$, less than minimum reporting level; --, no data]

\begin{tabular}{|c|c|c|c|c|c|c|c|c|c|}
\hline Date & Time & $\begin{array}{c}\text { Specific } \\
\text { conductance, } \\
\text { onsite } \\
(\mu \mathrm{S} / \mathrm{cm})\end{array}$ & $\begin{array}{c}\text { pH, } \\
\text { onsite } \\
\text { (standard } \\
\text { units) }\end{array}$ & $\begin{array}{c}\text { Calcium, } \\
\text { dissolved } \\
\text { (mg/L } \\
\text { as Ca) }\end{array}$ & $\begin{array}{c}\text { Mag- } \\
\text { nesium, } \\
\text { dissolved } \\
\text { (mg/L as } \\
\text { Mg) }\end{array}$ & $\begin{array}{c}\text { Sodium, } \\
\text { dissolved } \\
\text { (mg/L as } \\
\mathrm{Na})\end{array}$ & $\begin{array}{l}\text { Potassium, } \\
\text { dissolved } \\
\text { (mg/L as } \\
\text { K) }\end{array}$ & $\begin{array}{l}\text { Alkalinity, } \\
\text { lab } \\
(\mathrm{mg} / \mathrm{L} \text { as } \\
\left.\mathrm{CaCO}_{3}\right)\end{array}$ & $\begin{array}{c}\text { Sulfate, } \\
\text { dissolved } \\
(\mathrm{mg} / \mathrm{L} \text { as } \\
\left.\mathrm{SO}_{4}\right)\end{array}$ \\
\hline $09-06-95$ & 1120 & 2 & 6.6 & $<0.02$ & $<0.01$ & $<0.2$ & $<0.1$ & 1.2 & $<0.1$ \\
\hline $11-01-95$ & 1430 & 2 & -- & $<.02$ & $<.01$ & $<.2$ & $<.1$ & 1.1 & $<.1$ \\
\hline $04-19-96$ & 0800 & -- & -- & $<.02$ & $<.01$ & $<.2$ & $<.1$ & 1.6 & $<.1$ \\
\hline $06-20-96$ & 1200 & 2 & 6.7 & $<.02$ & $<.01$ & $<.2$ & $<.1$ & $<1$ & $<.1$ \\
\hline $08-21-96$ & 0830 & 1 & 5.8 & $<.02$ & $<.01$ & $<.2$ & $<.1$ & 1.2 & $<.1$ \\
\hline $10-24-96$ & 0830 & 3 & 5.8 & $<.02$ & $<.01$ & $<.2$ & $<.1$ & 1.5 & $<.1$ \\
\hline $04-14-97$ & 1030 & 2 & 6.1 & $<.02$ & $<.01$ & $<.2$ & $<.1$ & 1.7 & $<.1$ \\
\hline $05-30-97$ & 1115 & 1 & 6.1 & $<.02$ & $<.01$ & $<.2$ & $<.1$ & 1.3 & $<.1$ \\
\hline
\end{tabular}

\begin{tabular}{|c|c|c|c|c|c|c|c|}
\hline Date & $\begin{array}{c}\text { Chloride, } \\
\text { dissolved } \\
(\mathrm{mg} / \mathrm{L} \text { as } \mathrm{Cl})\end{array}$ & $\begin{array}{c}\text { Fluoride, } \\
\text { dissolved } \\
\text { (mg/L as F) }\end{array}$ & $\begin{array}{c}\text { Silica, } \\
\text { dissolved } \\
\text { (mg/L as } \\
\mathrm{SiO}_{2} \text { ) }\end{array}$ & $\begin{array}{c}\text { Nitrite, } \\
\text { dissolved } \\
(\mathrm{mg} / \mathrm{L} \text { as } \mathrm{N})\end{array}$ & $\begin{array}{c}\text { Nitrite plus } \\
\text { nitrate, dissolved } \\
(\mathrm{mg} / \mathrm{L} \text { as } \mathrm{N})\end{array}$ & $\begin{array}{c}\text { Ammonia, } \\
\text { dissolved } \\
\text { (mg/L as N) }\end{array}$ & $\begin{array}{c}\text { Phosphorus, } \\
\text { orthophosphate, } \\
\text { dissolved } \\
\text { (mg/L as P) }\end{array}$ \\
\hline $09-06-95$ & $<0.1$ & $<0.1$ & $<0.1$ & $<0.01$ & $<0.05$ & $<0.02$ & $<0.01$ \\
\hline $11-01-95$ & $<.1$ & $<.1$ & $<.1$ & $<.01$ & $<.05$ & $<.02$ & $<.01$ \\
\hline $04-19-96$ & $<.1$ & $<.1$ & $<.1$ & $<.01$ & $<.05$ & $<.02$ & $<.01$ \\
\hline $06-20-96$ & $<.1$ & $<.1$ & $<.1$ & $<.01$ & $<.05$ & .02 & $<.01$ \\
\hline $08-21-96$ & $<.1$ & $<.1$ & .2 & $<.01$ & $<.05$ & $<.02$ & $<.01$ \\
\hline $10-24-96$ & $<.1$ & $<.1$ & .1 & $<.01$ & $<.05$ & $<.02$ & $<.01$ \\
\hline $04-14-97$ & $<.1$ & $<.1$ & $<.1$ & $<.01$ & $<.05$ & $<.02$ & $<.01$ \\
\hline $05-30-97$ & $<.1$ & $<.1$ & $<.1$ & - & $\ldots$ & -- & - \\
\hline
\end{tabular}

\begin{tabular}{|c|c|c|c|c|c|c|c|}
\hline Date & $\begin{array}{c}\text { Arsenic, } \\
\text { total } \\
\text { recov- } \\
\text { erable } \\
\text { ( } \mu \mathrm{g} / \mathrm{L} \text { as } \mathrm{As})\end{array}$ & $\begin{array}{l}\text { Cadmium, } \\
\text { total recov- } \\
\text { erable } \\
(\mu \mathrm{g} / \mathrm{L} \text { as } \mathrm{Cd})\end{array}$ & $\begin{array}{c}\text { Copper, } \\
\text { total } \\
\text { recoverable } \\
(\mu \mathrm{g} / \mathrm{L} \text { as } \mathrm{Cu})\end{array}$ & $\begin{array}{c}\text { Iron, } \\
\text { total } \\
\text { recoverable } \\
(\mu \mathrm{g} / \mathrm{L} \text { as } \mathrm{Fe})\end{array}$ & $\begin{array}{c}\text { Lead, } \\
\text { total } \\
\text { recoverable } \\
(\mu \mathrm{g} / \mathrm{L} \text { as } \mathrm{Pb})\end{array}$ & $\begin{array}{c}\text { Manganese, } \\
\text { total recov- } \\
\text { erable } \\
(\mu \mathrm{g} / \mathrm{L} \text { as } \mathrm{Mn})\end{array}$ & $\begin{array}{c}\text { Zinc, } \\
\text { total } \\
\text { recoverable } \\
(\mu \mathrm{g} / \mathrm{L} \text { as } \mathrm{Zn})\end{array}$ \\
\hline $09-06-95$ & $<1$ & $<1$ & $<1$ & $<10$ & $<1$ & $<10$ & $<10$ \\
\hline $11-01-95$ & $<1$ & $<1$ & 2 & $<10$ & $<1$ & $<10$ & $<10$ \\
\hline $04-19-96$ & $<1$ & $<1$ & $<1$ & $<10$ & $<1$ & $<10$ & $<10$ \\
\hline $06-20-96$ & $<1$ & $<1$ & $<1$ & $<10$ & $<1$ & $<10$ & $<10$ \\
\hline $08-21-96$ & $<1$ & $<1$ & $<1$ & $<10$ & $<1$ & $<10$ & $<10$ \\
\hline $10-24-96$ & $<1$ & $<1$ & $<1$ & $<10$ & $<1$ & $<10$ & $<10$ \\
\hline $04-14-97$ & $<1$ & $<1$ & $<1$ & $<10$ & $<1$ & $<10$ & $<10$ \\
\hline $05-30-97$ & $<1$ & $<1$ & $<1$ & $<10$ & $<1$ & $<10$ & $<10$ \\
\hline
\end{tabular}


Table 5. Montana water-quality maximum guideline concentrations for selected water uses

[Abbreviations: $\mu \mathrm{g} / \mathrm{L}$, micrograms per liter; $\mathrm{mg} / \mathrm{L}$, milligrams per liter. Symbol: --, no guideline concentration has been adopted]

\begin{tabular}{|c|c|c|c|c|c|}
\hline \multirow{3}{*}{ Constituent } & \multicolumn{5}{|c|}{ Maximum guideline concentration for indicated water use } \\
\hline & \multirow{2}{*}{$\begin{array}{c}\text { Human } \\
\text { consumption }\end{array}$} & \multicolumn{2}{|c|}{ Aquatic life $^{1,2}$} & \multirow{2}{*}{ Irrigation $^{3}$} & \multirow{2}{*}{$\begin{array}{l}\text { Livestock } \\
\text { watering }\end{array}$} \\
\hline & & Acute & Chronic & & \\
\hline \multicolumn{6}{|c|}{ TRACE ELEMENTS } \\
\hline Arsenic $(\mu \mathrm{g} / \mathrm{L})$ & 18 & 360 & 190 & 100 & 200 \\
\hline Cadmium $(\mu g / L)$ & 5 & 43.9 & ${ }^{4} 1.1$ & 50 & 50 \\
\hline Copper $(\mu \mathrm{g} / \mathrm{L})$ & 1,000 & ${ }^{4} 18$ & ${ }^{4} 12$ & 5,000 & 500 \\
\hline Iron $(\mu \mathrm{g} / \mathrm{L})$ & 300 & -- & 1,000 & 20,000 & - \\
\hline Lead $(\mu \mathrm{g} / \mathrm{L})$ & 15 & ${ }^{4} 82$ & 43.2 & 10,000 & 100 \\
\hline Manganese $(\mu \mathrm{g} / \mathrm{L})$ & 50 & -- & - & 10,000 & - \\
\hline $\operatorname{Zinc}(\mu \mathrm{g} / \mathrm{L})$ & - & ${ }^{4} 120$ & ${ }^{4} 110$ & -- & - \\
\hline \multicolumn{6}{|c|}{ NUTRIENTS } \\
\hline Nitrogen, ammonia $(\mathrm{mg} / \mathrm{L})^{5}$ & -- & 6.15 & 6.02 & -- & - \\
\hline Nitrogen, nitrite $(\mathrm{mg} / \mathrm{L})^{5}$ & 1 & - & - & -- & 10 \\
\hline Nitrogen, nitrite plus nitrate $(\mathrm{mg} / \mathrm{L})^{5}$ & 10 & -- & -- & -- & 100 \\
\hline
\end{tabular}

${ }^{1}$ Montana Department of Environmental Quality (1995).

${ }^{2}$ Aquatic-life standards refer to total-recoverable concentrations.

${ }^{3}$ Montana Department of Health and Environmental Science (1986, p. 8)

${ }^{4}$ Concentration listed for the protection of aquatic life is based on a water hardness of 100 milligrams per liter as calcium carbonate. Standards for cadmium, copper, lead, and zinc vary with water hardness (see fig. 2).

${ }^{5} \mathrm{~A}$ plant nutrient, excessive amounts of which may cause violations of Administrative Rules of Montana (ARM) 16.20.633.(1)(e).

${ }^{6}$ Concentration listed for the protection of aquatic life is based on a pH between 8 and 9 and a temperature less than 15 degrees Celsius. 